\title{
Spleen mediates a distinct hematopoietic progenitor response supporting tumor-promoting myelopoiesis
}

\author{
Chong Wu, ${ }^{1,2}$ Huiheng Ning, ${ }^{2}$ Mingyu Liu, ${ }^{2}$ Jie Lin, ${ }^{2}$ Shufeng Luo, ${ }^{2}$ Wenjie Zhu, ${ }^{1}$ Jing Xu, ${ }^{1}$ Wen-Chao Wu, ${ }^{1}$ Jing Liang, ${ }^{3}$ \\ Chun-Kui Shao, ${ }^{3}$ Jiaqi Ren, ${ }^{4}$ Bin Wei, ${ }^{4}$ Jun Cui, ${ }^{2}$ Min-Shan Chen, ${ }^{1}$ and Limin Zheng ${ }^{1,2}$ \\ 'State Key Laboratory of Oncology in South China, Collaborative Innovation Center for Cancer Medicine, Sun Yat-sen University Cancer Center, Guangzhou, China. ${ }^{2}$ Key Laboratory of Gene Engineering of \\ the Ministry of Education, Sun Yat-sen University, Guangzhou, China. ${ }^{3}$ Department of Pathology, Guangdong Key Laboratory of Liver Disease Research, The Third Affiliated Hospital, Sun Yat-sen University, \\ Guangzhou, China. ${ }^{4}$ State Key Laboratory of Virology, Wuhan Institute of Virology, Chinese Academy of Sciences, Wuhan, China.
}

\begin{abstract}
Cancer progression is associated with alterations of intra- and extramedullary hematopoiesis to support a systemic tumorpromoting myeloid response. However, the functional specialty, mechanism, and clinical relevance of extramedullary hematopoiesis (EMH) remain unclear. Here, we showed that the heightened splenic myelopoiesis in tumor-bearing hosts was not only characterized by the accumulation of myeloid precursors, but also associated with profound functional alterations of splenic early hematopoietic stem/progenitor cells (HSPCs). With the distinct capability to produce and respond to granulocyte-macrophage CSF (CM-CSF), these splenic HSPCs were "primed" and committed to generating immunosuppressive myeloid cells. Mechanistically, the CCL2/CCR2 axis-dependent recruitment and the subsequent local education by the splenic stroma were critical for eliciting this splenic HSPC response. Selective abrogation of this splenic EMH was sufficient to synergistically enhance the therapeutic efficacy of immune checkpoint blockade. Clinically, patients with different types of solid tumors exhibited increased splenic HSPC levels associated with poor survival. These findings reveal a unique and important role of splenic hematopoiesis in tumor-associated myelopoiesis.
\end{abstract}

\section{Introduction}

Cancer is associated with a profound myeloid response resulting in the expansion of tumor-associated myeloid cells, including myeloid-derived suppressor cells (MDSCs), neutrophils, and macrophages, to promote disease progression (1-3). These myeloid cells modulate adaptive immune responses against tumors (3-5), enhance cancer cell stemness $(6,7)$, and create conditions that support angiogenesis, invasion, and metastasis (2, 3, 8-10). However, these cells are generally short-lived (11) and must be continuously replenished throughout the progression of cancer. Therefore, to guarantee a sufficient pool of myeloid cells, cancer interferes with hematopoietic stem and progenitor cell (HSC and HPC, or HSPC combined) activity and reroutes the host's hematopoiesis to generate cells of myeloid lineages with profound tumor-promoting functions $(12,13)$. These findings are consistent with clinical observations that the progression of different types of solid tumors is associated with an increased peripheral neutrophil-to-lymphocyte ratio $(14,15)$ and an elevated level of circulating granulocyte-macrophage progenitors (GMPs) (16). Thus, defining the nature and characteristics of the altered hematopoiesis in cancer is crucial for understanding the systemic tumor-promoting myeloid response.

In the steady state, the postnatal development of blood cell lineages primarily occurs in BM. However, recent studies have revealed that tumor-derived factors, including granulo-

Conflict of interest: The authors have declared that no conflict of interest exists Submitted: October 12, 2017; Accepted: May 10, 2018.

Reference information: / Clin Invest. 2018;128(8):3425-3438.

https://doi.org/10.1172/JCI97973. cyte-macrophage CSF (GM-CSF) (17-19), granulocyte-CSF (G-CSF) (12), and peptide hormone angiotensin II (20), mediate systemic deviation of hematopoiesis, not only in the BM but also in extramedullary tissues such as the spleen (12, 20-22). Yet, it is unclear whether different sites of hematopoiesis are established for distinct purposes. In particular, little is known about the functional differences between intra- and extramedullary HSPCs and between the myeloid cells derived from the $\mathrm{BM}$ and spleen of tumor-bearing hosts (22). Also, it remains to be addressed whether and how the spleen could selectively accumulate HSPC subsets in a population- and organ-specific manner and what roles the local environmental factors play in regulating the functional properties of splenic HSPCs. Identifying the specific mechanisms regulating tumor-induced splenic extramedullary hematopoiesis (EMH) might provide a novel strategy for anticancer therapy.

In the present study, we investigated the function, mechanism, and clinical relevance of cancer-induced splenic EMH. We found that splenic EMH is not a mere quantitative supplement to $\mathrm{BM}$ hematopoiesis in tumor-bearing hosts, but rather has a unique and significant function by selectively amplifying a specific HSPC response committed to generating immunosuppressive myeloid cells.

\section{Results}

Myeloid-biased splenic early HSPCs in tumor-bearing mice. Hepatocellular carcinoma (HCC) represents a type of cancer that is associated with negligible or low levels of systemic myeloid differentiation cytokines but evident extramedullary HSPC activity (16). To inves- 
A

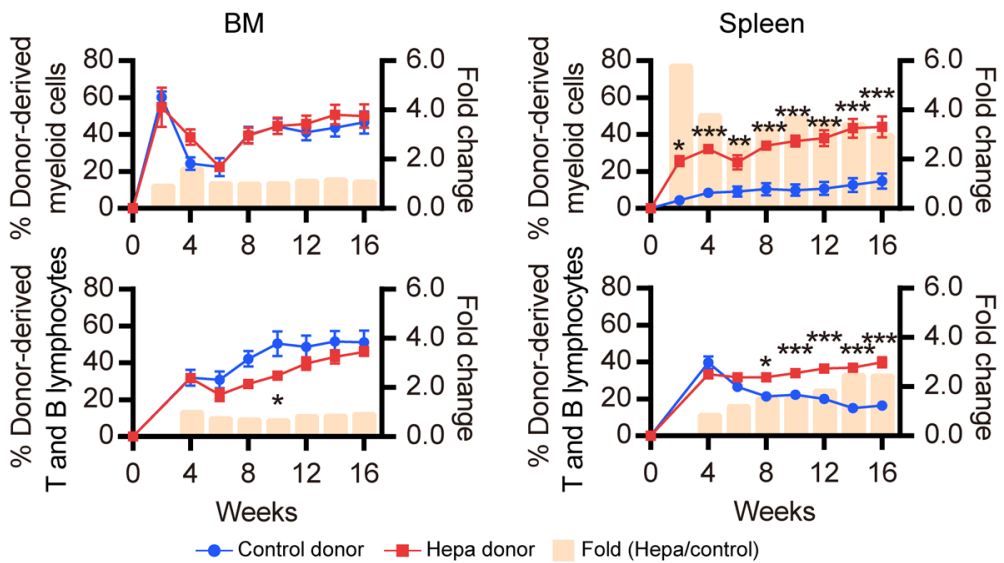

B

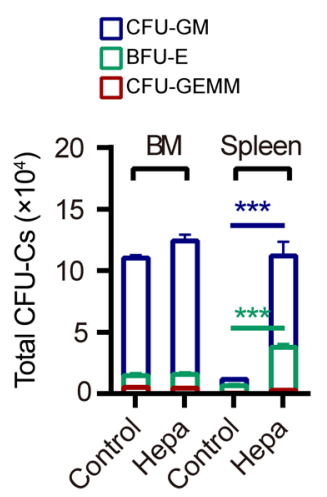

C

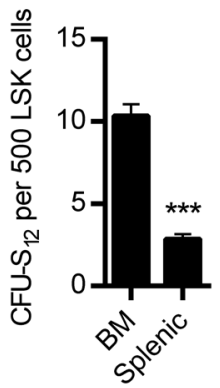

D

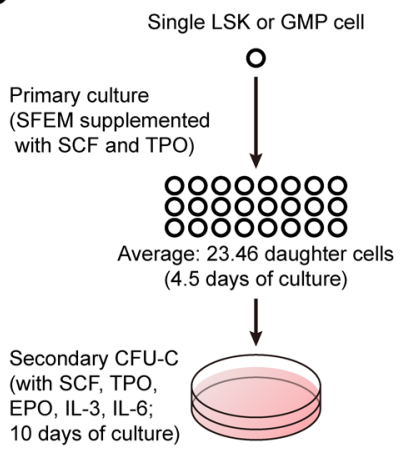

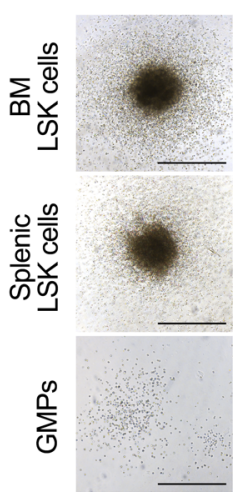
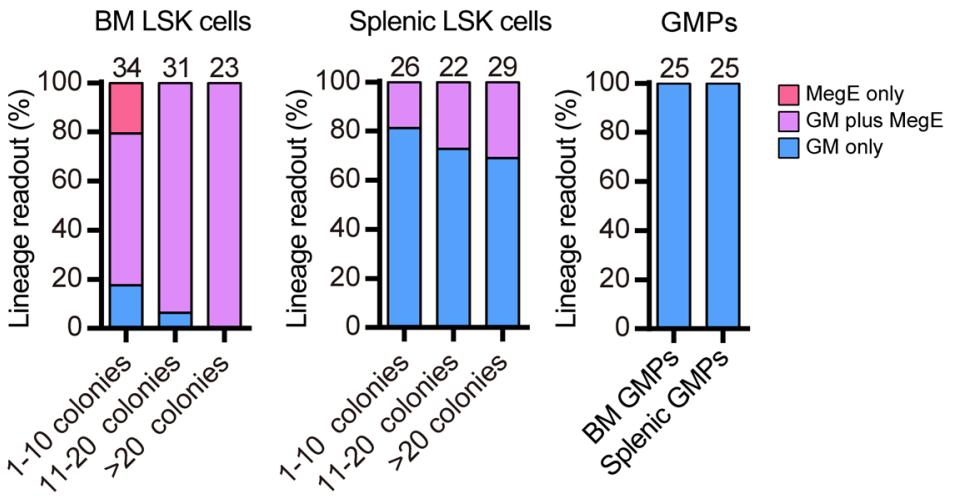

Figure 1. The myeloid-biased differentiation of splenic early HSPCs from Hepa mice. (A) Analysis of peripheral blood myeloid cell (CD11b+Gr-1+) and lymphocyte (CD3 ${ }^{+}$T and B220 $B$ cells) reconstitution chimerisms in recipient mice (BM, $n=7$ mice per group; spleen, $n=9$ mice per group). ${ }^{*} P<0.05$, ${ }^{* *} P<0.01$, and ${ }^{* *} P<0.001$, by 2-way ANOVA with Bonferroni's correction. (B) Quantification of CFU-C activity in BM and spleens from control ( $n=7$ ) and Hepa $(n=8)$ mice. ${ }^{* *} P<0.001$, by Student's $t$ test. BFU-E, burst-forming unit-erythroid. (C) CFU-S activity of 500 BM-derived ( $n=15$ recipients) or splenic ( $n=21$ recipients) LSK cells from Hepa mice. ${ }^{* *} P<0.001$, by Student's $t$ test. (D) Scheme of the 2 -step single-cell colony-forming assay, representative colonies, and the percentage of different types of lineage readouts of the secondary CFU-C. Scale bars: $500 \mu \mathrm{m}$. Numbers above the columns represent the sample size of the initiating single cells in each group. Results are shown as the mean \pm SEM of mice in each group (A-C). Data are from 2 experiments (A and $\mathbf{B}$ ) or 3 experiments, with cells pooled from 6 to 10 mice (C and $\mathbf{D})$.

tigate the cancer-associated EMH in such tumor settings, we used an orthotopic Hepa1-6 hepatoma mouse model (hereafter referred to as Hepa mice), which reproduces many features of human HCC $(23,24)$. In this model, the spleen was the major site of EMH (Supplemental Figure 1, A and B; supplemental material available online with this article; https://doi.org/10.1172/JCI97973DS1). With slight splenomegaly, the spleens of Hepa mice accumulated both $\mathrm{Lin}^{\mathrm{lo} /}$ Sca- ${ }^{+}{ }^{+}$-Kit ${ }^{\text {hi }}$ (LSK) and Lin $^{\text {lo/-Sca- }}{ }^{-}{ }^{-}$-Kit ${ }^{\text {hi }}$ (LK) HSPCs in a progression-dependent manner (Supplemental Figure 1, C-E) and at levels comparable to those detected in other transplanted or genetic mouse tumor models (Supplemental Figure 1, F and G).

To describe and compare the cancer-associated hematopoietic alterations in $\mathrm{BM}$ and spleen, we initially performed competitive reconstitution experiments. BM from Hepa mice reconstituted myeloid cells and lymphocytes at levels similar to those of its control counterpart (Figure 1A). In contrast, the transfer of splenocytes from Hepa mice led to a rapid and more robust reconstitution compared with that seen in control spleen, with a myelopoiesis preference (Figure 1A). The fold changes of myeloid reconstitution peaked 2 weeks after transplanta- tion, showing 6-fold greater chimerisms compared with control spleen and gradually decreased afterwards. In accordance with the boost of myeloid cell reconstitution, CFU assays showed a 15-fold increase in CFU-GM in the spleens of Hepa mice (Figure 1B), whereas the enrichment of multi-lineage CFU-granulocyte/erythrocyte/macrophage/megakaryocyte (CFU-GEMM) was insignificant. These findings suggested that the spleens of Hepa mice accommodated a myeloid-biased hematopoiesis facilitating rapid myeloid immune cell generation.

Heightened splenic myelopoiesis in cancer has been linked to the accumulation of multiple HSPC populations $(13,20,21)$, but the functional preference of early HSPCs, the LSK cells, in the spleen of the tumor-bearing host remains unclear. These LSK cells are highly heterogeneous and contain various HSC and HPC subpopulations with different lineage potential (25-27). Although BM and splenic LSK cells from Hepa mice differentiated into $\mathrm{Fc}_{\mathrm{R}} \mathrm{R}^{\mathrm{lo}}$ $\mathrm{CD}_{4} 4^{+}$common myeloid progenitors (CMPs) and $\mathrm{Fc} \gamma \mathrm{R}^{\mathrm{hi}} \mathrm{CD} 34^{+}$ GMPs at similar kinetics in vitro (Supplemental Figure $1, \mathrm{H}$ and I), LSK cells from the spleen produced markedly fewer trilineage splenic CFU in vivo (CFU-S ${ }_{12}$ ) (Figure 1C), suggesting a reduced 
A

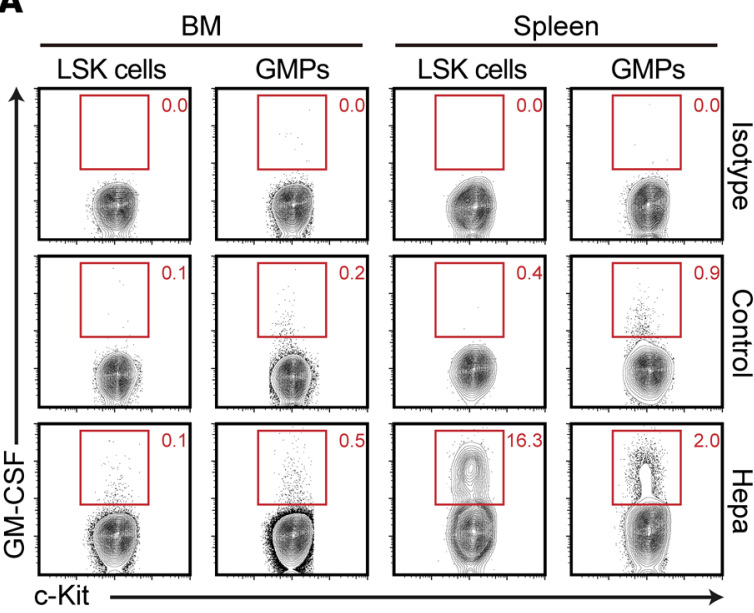

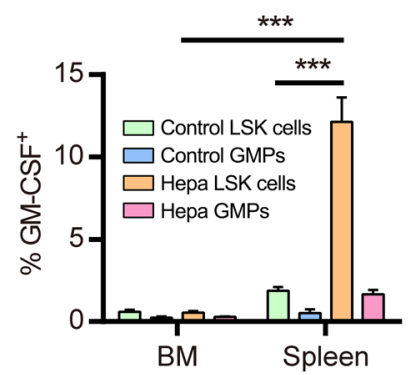

B

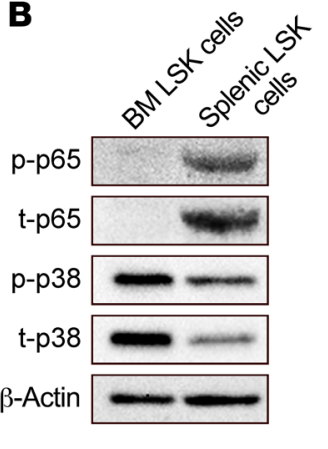

C

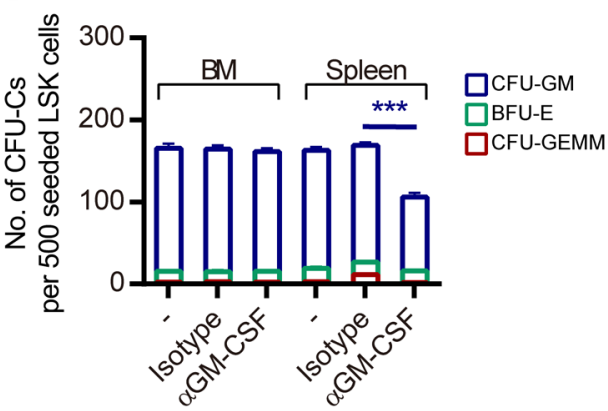

D

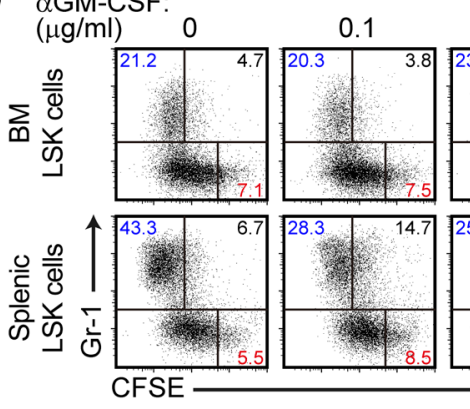

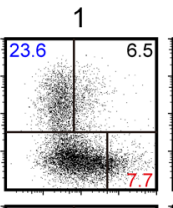
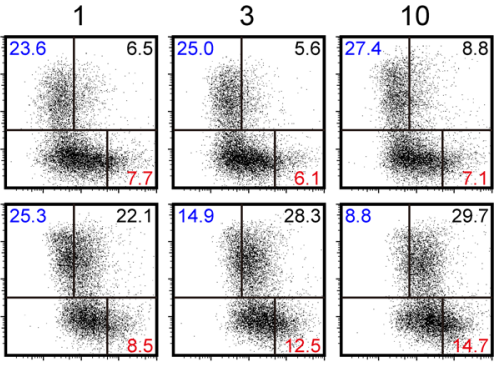

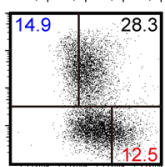

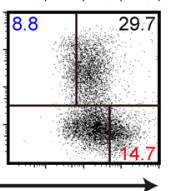

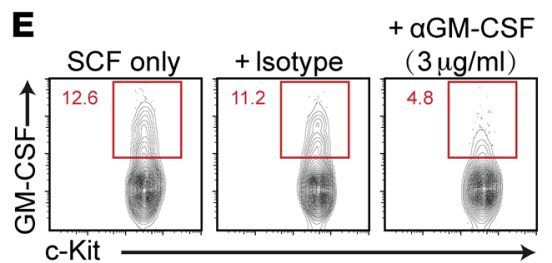

Figure 2. Accumulation of GM-CSF-expressing LSK cells in the spleens of tumor-bearing mice. (A) Endogenous GM-CSF expression in freshly isolated LSK cells and GMPs (FcyR $\left.{ }^{\text {hicD34 }}{ }^{+} \mathrm{LK}\right)\left(n=6\right.$ per group). ${ }^{* * *} P<0.001$, by 2 -way ANOVA followed by Dunnett's test. (B) Immunoblot for NF- $K B$ p65 and MAPK p38 activation in LSK cells isolated from BM and spleens of Hepa mice. p, phosphorylated; $t$, total. (C) Clonogenic ability of 500 BM or splenic LSK cells isolated from Hepa mice ( $n=6$ per group) in the methylcellulose-based assay. Anti-GM-CSF ( $\alpha$ GM-CSF): $3 \mu \mathrm{g} / \mathrm{ml}$. ${ }^{* * *} P<0.001$, by 2 -way ANOVA followed by Dunnett's test. (D) LSK cells were CFSE stained and cultured for 5 days in serum-free medium supplemented with SCF and the indicated concentration of anti-CM-CSF Abs in the cultures. The proliferation and differentiation HSPCs into myeloid cells are shown by CFSE dilution. (E) GM-CSF expression in LSK cells was examined after 24 hours of the cultures described in $\mathbf{D}$. Numbers in the flow cytometric plots indicate the proportions of gated cells (A, D, and $\mathbf{E}$ ). Results are shown as the mean \pm SEM for mice in each group ( $\mathbf{A}$ and $\mathbf{C})$. Data are from 2 experiments (A), 3 experiments with cells pooled from 6 to 10 mice $(\mathbf{C})$, or representative of at least 3 experiments $(\mathbf{B}, \mathbf{D}$, and $\mathbf{E})$.

proportion of multipotent HSCs (28). To corroborate the differentiation potential of splenic LSK cells at the single-cell level, we adopted a 2-step colony-forming assay (29). The daughter cells from more than $85 \%$ single BM LSK cells simultaneously formed both GM-type and megakaryocyte-erythrocyte-type (MegE-type) colonies, indicating that most BM LSK cells were multipotent (Figure 1D). In stark contrast, the daughter cells from more than $70 \%$ single splenic LSK cells formed GM-type colonies only, suggesting that the majority of splenic LSK cells were myeloid immune cell restricted. These findings indicate that the heightened splenic myelopoiesis in tumor-bearing mice is not only characterized by the accumulation of HSPCs, but is also associated with a significant myeloid skew within the LSK cell population.
Accumulation of GM-CSF-expressing LSK cells in the spleens of tumor-bearing mice. Emerging evidence has suggested that the biased lineage potential of LSK cells may be associated with their altered cytokine production capacity (30). Along this line, we found that a significantly higher proportion of splenic LSK cells from Hepa mice expressed GM-CSF, an important myeloid differentiation cytokine (Figure 2A). The enhanced GM-CSF expression was associated with upregulation of NF- $\mathrm{KB}$ activation and downregulation of p38 MAPK activation (Figure 2B; see complete unedited blots in the supplemental material). These GM-CSFexpressing HSPCs were commonly present in the spleens of tumor-bearing mice, including in another hepatoma model induced by $\mathrm{N}$-nitrosodiethylamine (DEN) and $\mathrm{CCl}_{4}$, a transplant- 
A

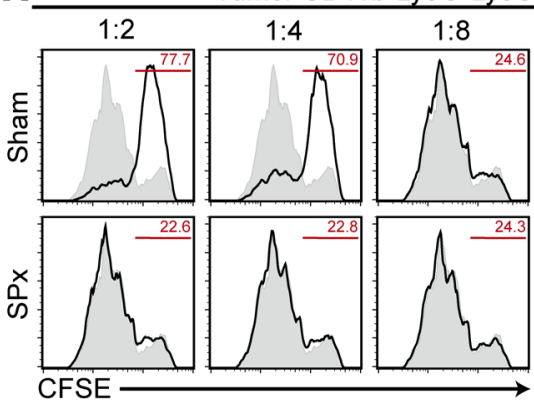

Tumor CD11 b+Ly6G+Ly6C ${ }^{10}(\mathrm{PMN}-$ like) cells

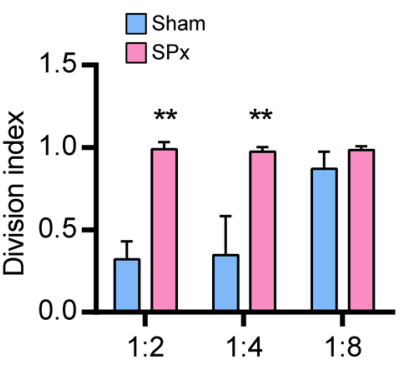

E
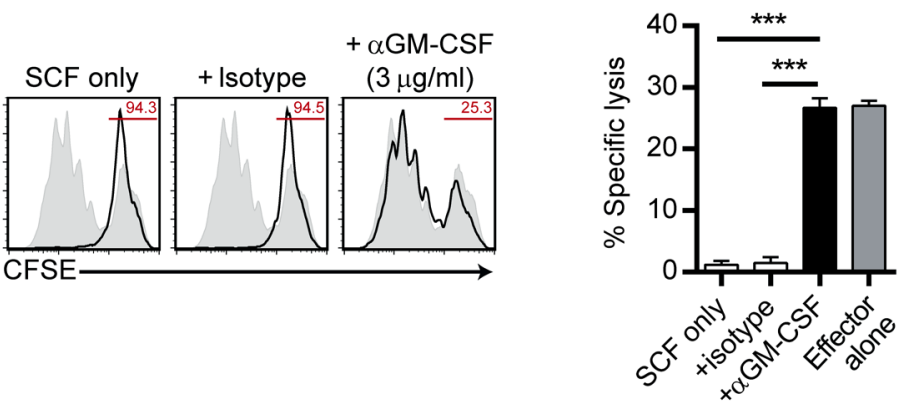

B

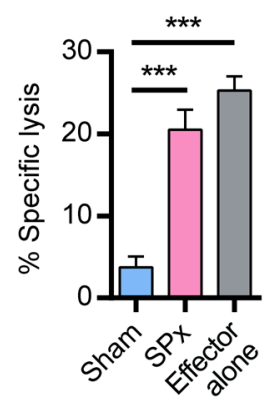

C

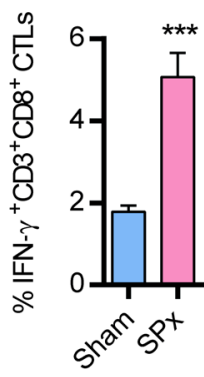

D

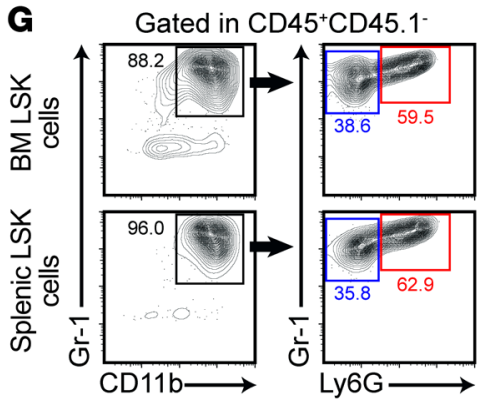

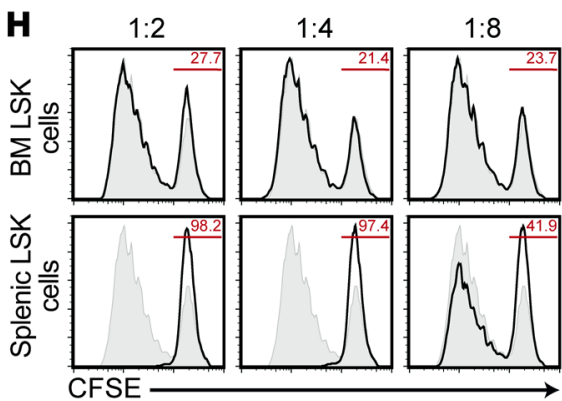

$\mathbf{F}$

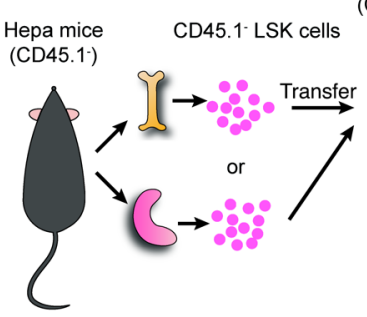

Tumor-free ecipient mice (CD45.1 $\left.1^{+}\right)$

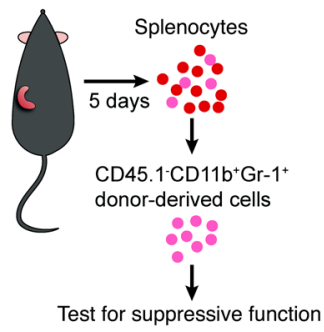

Figure 3. Endogenous GM-CSF drives splenic HSPCs to produce myeloid suppressor cells. (A and B) Immunosuppressive effect of tumor-infiltrating CD11b+Ly6C ${ }^{+}$Ly6 ${ }^{10}$ PMN-like myeloid cells on anti-CD3- and anti-CD28-stimulated T cell proliferation (A) and on the cytotoxic activity of CD8 ${ }^{+}$T cells elicited by antigen-specific stimulation in vitro (B). Hepa mice were subjected to sham surgery or splenectomy (SPX). ${ }^{* *} P<0.01$ and ${ }^{* * *} P<0.001$, by 2 -way ANOVA with Bonferroni's correction. (C) Frequencies of tumor-infiltrating IFN- $\gamma^{+}$CD ${ }^{+}$CD8 ${ }^{+}$CTLs in Hepa mice subjected to sham surgery or splenectomy. ${ }^{* * *} P<0.001$, by Student's $t$ test. ( $\mathbf{D}$ and $\mathbf{E}$ ) Splenic LSK cells were cultured in serum-free medium with SCF for 4 days. The Gr-1 ${ }^{+}$myeloid descendants were isolated and tested for their suppressive effect on anti-CD3- and anti-CD28-stimulated T cell proliferation at a ratio of 1:4 (D) and on the antigen-induced cytotoxic activity of CD8 ${ }^{+} T$ cells $(\mathbf{E}){ }^{* * *} P<0.001$, by 2-way ANOVA with Bonferroni's correction. (F) Cartoon depicting the adoptive transfer of CD45.1 BM or splenic LSK cells into the spleens of CD45.1+ tumor-free recipient mice. (G-I) Representative phenotype (G) and suppressive activity (H and I) of donor-derived $\mathrm{CD} 11 \mathrm{~b}^{+} \mathrm{Gr}-1^{+}$myeloid cells retrieved from the spleens of recipient mice ( $n=3$ per group) as described in $\mathbf{F} .{ }^{* *} P<0.01$ and ${ }^{* * *} P<0.001$, by 2-way ANOVA with Bonferroni's correction. Results are shown as the mean \pm SEM (A-C, E, H, and I). CFSE+ splenocyte proliferation when cocultured with myeloid descendant cells at the indicated ratios (solid lines) or cultured alone (shaded) is shown (A, $\mathbf{D}$, and $\mathbf{H})$. Numbers in the flow cytometric plots indicate the proportions of gated cells (A, D, G, and $\mathbf{H})$. Data are representative of 2 experiments (A-C, $n=3$ per group in each experiment) or 3 experiments, with cells pooled from 6 to 10 mice (D-I).

able melanoma model, and an intestinal neoplasia model caused by the Apc gene mutation (Supplemental Figure 2A). However, these cells were rarely detected in the $\mathrm{BM}$, in the control spleen (Figure 2A), or in an EMH model induced by repeated bleeding (Supplemental Figure 2A). Moreover, we found that the level of GM-CSF was not increased in the serum or in the splenic microenvironment of Hepa mice (Supplemental Figure 2, B-E) and that another important cytokine, G-CSF, was also not increased in this setting (Supplemental Figure 2F). These findings suggested that the endogenous GM-CSF signal might be functionally significant for splenic LSK HSPC differentiation.
To confirm the function of endogenous GM-CSF, we found that neutralizing Abs against GM-CSF impaired CFU-GM activity (with stem cell factor [SCF], IL-3, IL-6, and erythropoietin in the methylcellulose-based culture) of splenic, but not BM, LSK cells (Figure $2 \mathrm{C}$ ). In addition, neutralizing the endogenous GM-CSF in an essential HSPC culture condition (serum-free medium supplemented with SCF only) attenuated the proliferation of splenic LSK cells and their differentiation into myeloid cells in a dose-dependent manner (Figure 2D). These data suggested an important role of this endogenous signal in mediating LSK cell myelopoiesis in both simple and complex cytokine environments. Moreover, the presence of 
A

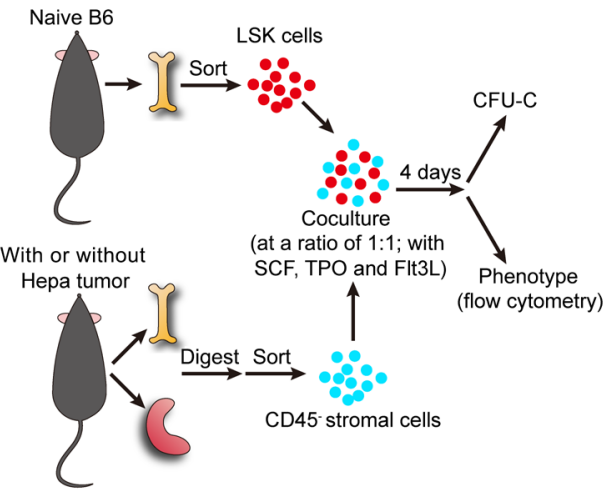

D Naive B6 (CD45.1')

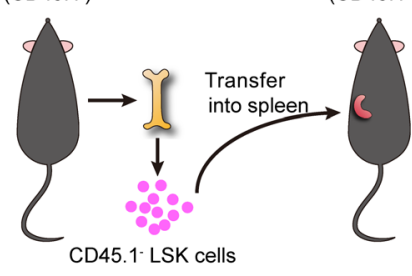

B

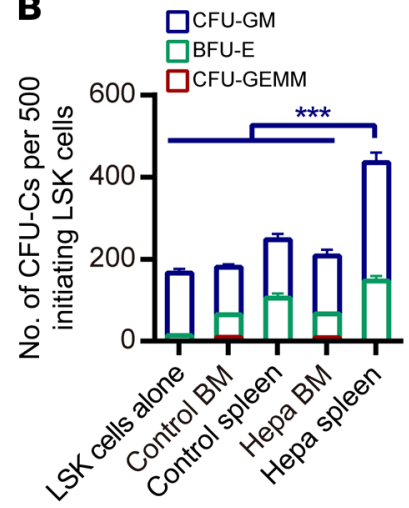

C LSK cells

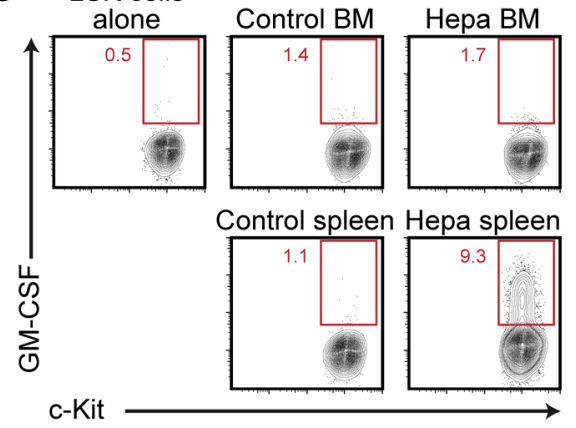

E
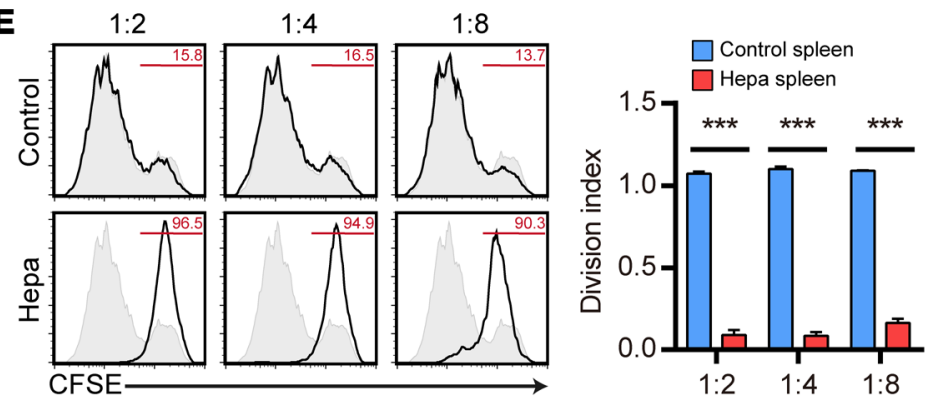

$\mathbf{F}$

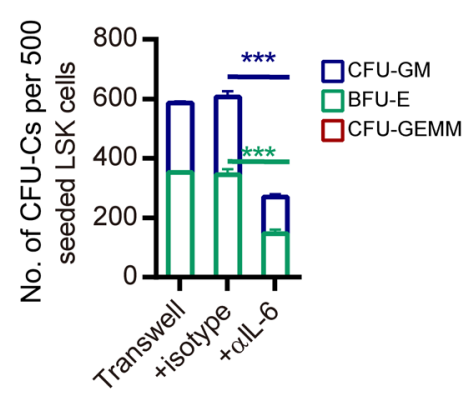

G

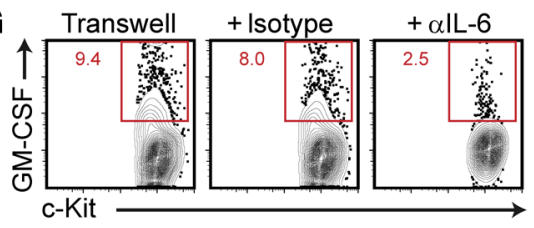

H Transwell

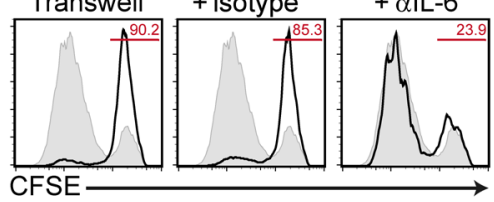

Figure 4. Splenic stroma of tumor-bearing host supports LSK cells in the production of MDSCs. (A) Scheme of the coculture experiments. (B and C) CFU-C activity (B) and endogenous CM-CSF expression (C) of BM-derived naive LSK cells after coculture with CD45- stromal cells from the indicated tissues. ${ }^{* * *} P<0.001$, by 2-way ANOVA followed by Dunnett's test. (D) Cartoon depicting the adoptive transfer of CD45.1- BM-derived naive LSK cells into the spleens of $C D 45.1^{+}$tumor-free or tumor-bearing recipient mice. (E) Suppressive activity of donor-derived CD11 b+Gr-1+ myeloid cells retrieved from the spleens of the recipient mice described in $\mathbf{D}$. ${ }^{* *} P<0.001$, by 2 -way ANOVA with Bonferroni's correction. ( $\mathbf{F}$ and $\left.\mathbf{G}\right)$ CFU-C activity (F) and endogenous CM-CSF expression (C) of BM-derived naive LSK cells after coculture in Transwells with splenic stromal cells from Hepa mice with the indicated Abs (1 $\mu \mathrm{g} / \mathrm{ml})$ in the cultures. ${ }^{* * *} P<0.001$, by 2-way ANOVA with Bonferroni's correction. (H) After 4 days of coculture in Transwells as described in $\mathbf{F}$ and $\mathbf{G}$, Lin ${ }^{-}$HSPCs were isolated and transferred into serum-free medium supplemented with SCF only. After another 3 days of culture, the Gr- $1^{+}$myeloid descendants were isolated and tested for their suppressive activity. CFSE+ splenocyte proliferation when cocultured with myeloid descendant cells at the indicated ratios (solid lines) or cultured alone (shaded) is shown ( $\mathbf{E}$ and $\mathbf{H}$ ). Numbers in the flow cytometric plots indicate the proportions of gated cells (C, $\mathbf{E}, \mathbf{G}$, and $\mathbf{H}$ ). Data are shown as the mean \pm SEM of 3 experiments, with cells pooled from 6 to 8 mice (B, E, and $\mathbf{F}$ ) or are representative of 3 experiments, with cells pooled from 6 to 8 mice (C, $\mathbf{G}$, and $\mathbf{H})$.

anti-GM-CSF Abs in the culture also attenuated the intracellular GM-CSF expression of these splenic LSK cells, suggesting a positive feedback of this endogenous GM-CSF signal (Figure 2E). Together, these findings indicate that a significant fraction of splenic LSK cells upregulates the GM-CSF expression, thereby potentiating their myeloid differentiation, which may represent a distinct stressinduced HSPC response in tumor-bearing mice.

Endogenous GM-CSF drives splenic HSPCs to generate myeloid suppressors. We next investigated whether and how this splenic HSPC response contributed to the immunosuppressive function of tumor-associated myeloid cells. To evaluate the over- all effect of splenic EMH, we first performed splenectomy. We found that splenectomy did not change the frequencies or distribution of tumor myeloid cells (Supplemental Figure 3, $\mathrm{A}$ and $\mathrm{B})$. However, it did abolish the suppressive activity of

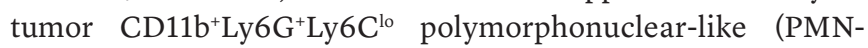
like) cells (or tumor PMN-MDSCs) (Figure 3, A and B), which was the major suppressive MDSC subset in Hepa tumors (Supplemental Figure 3, C and D). The abrogation of splenic EMH by splenectomy impaired the suppressive effect of tumor-infiltrating PMN-like cells on the proliferation and antigen-induced cytotoxic activity of $\mathrm{T}$ cells (Figure 3 , A and B). We observed 
A

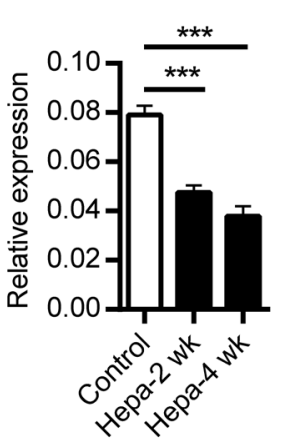

B $\quad \mathrm{C} / 2$

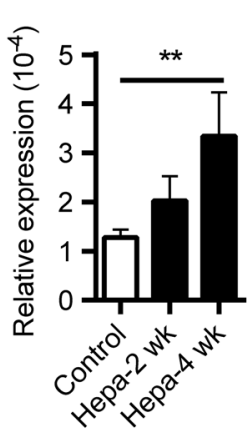

C

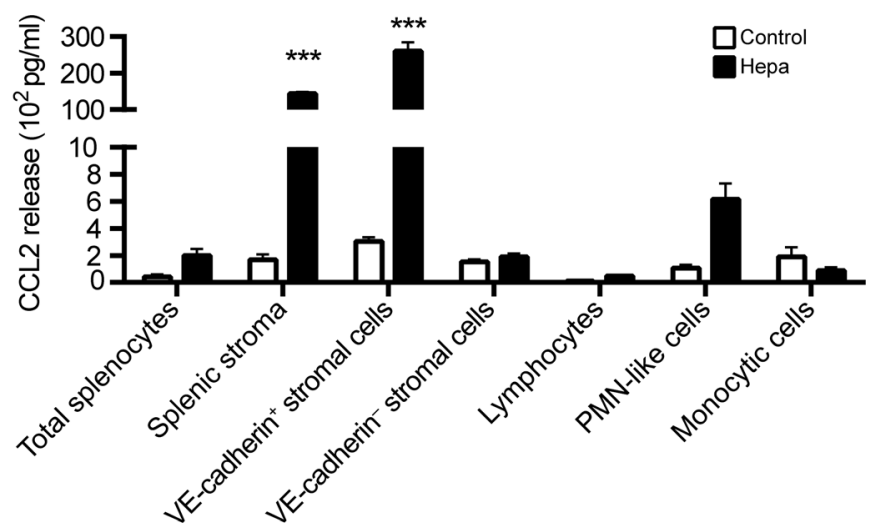

D

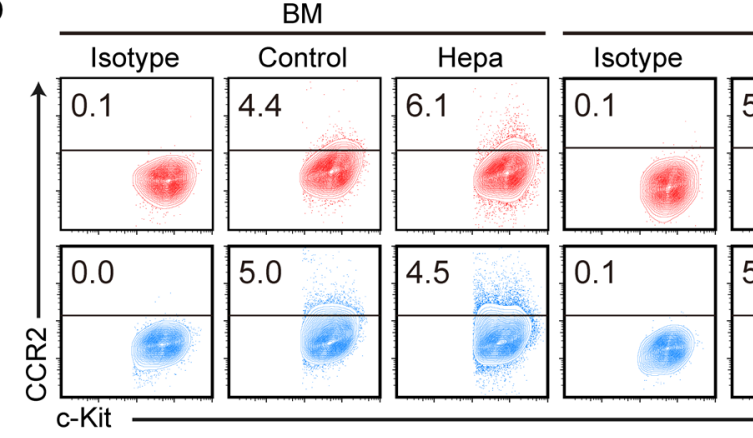

Spleen

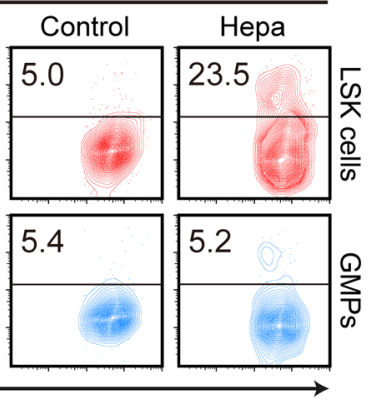

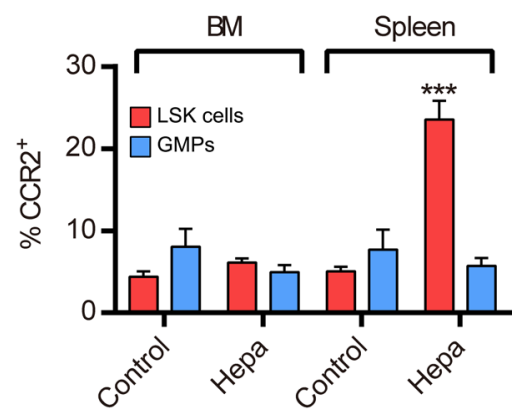

Figure 5. Enhanced CCL2/CCR2 signaling in the spleens of Hepa mice. (A and B) Splenic $C x c / 12$ (A) and $C c / 2$ (B) mRNA expression levels. Values are relative to Actb mRNA expression ( $n=6$ per group). ${ }^{* *} P<0.01$ and ${ }^{* * *} P<0.001$, by 1 -way ANOVA followed by Dunnett's test. (C) CCL2 release was measured by ELISA in the supernatants of 48 -hour cultures of the indicated cell populations isolated from the spleens of control or Hepa mice $(n=3$ per group, evaluated in triplicate). ${ }^{* *} P<0.001$, by 2-way ANOVA followed by Bonferroni's test. (D) Flow cytometric analysis of surface CCR2 expression on BM and splenic LSK and GMP cells from control $(n=7)$ and Hepa mice $(n=6)$. Numbers in the cytometric plots indicate the proportions of the gated cells. ${ }^{* *} P<0.001$, by 2 -way ANOVA followed by Tukey's test. Data are representative of at least 2 experiments and presented as the mean \pm SEM of the mice in each group (A-D).

that splenectomy suppressed the expression of arginase 1 (Arg1) in tumor-infiltrating PMN-like cells (Supplemental Figure 3, E-G; see complete unedited blots in the supplemental material), which was associated with the inhibition of STAT3 activation (Supplemental Figure 3G). In addition, splenectomy increased the frequencies of tumor-infiltrating IFN- $\gamma^{+}$ $\mathrm{CD}^{+} \mathrm{CD}^{+}$cytotoxic $\mathrm{T}$ lymphocytes (CTLs) by 2-fold (Figure $3 \mathrm{C}$ ). These findings suggested an important role of splenic $\mathrm{EMH}$ in mediating the suppressive myeloid response.

GM-CSF is a critical regulator of MDSC generation and activation $(8,19)$. However, it has been established that de novo induction of a naive progenitor/precursor cell to generate fully functional MDSCs requires at least 2 signals $(31,32)$. GM-CSF alone failed to induce $\mathrm{BM}$ progenitors from Hepa mice to produce functional $\mathrm{CD}_{11 \mathrm{~b}}{ }^{+} \mathrm{Gr}-1^{+} \mathrm{MDSC}$ in vitro (Supplemental Figure 3, $\mathrm{H}$ and I), consistent with a previous report (17). In contrast, GM-CSF effectively induced splenic LSK and GMP cells to generate myeloid cells with potent immunosuppressive activity in both serum-free and complete media (Supplemental Figure 3, H and I). These data suggested that the splenic HSPCs from Hepa mice, but not their BM phenotypic counterparts, included "primed" progenitors of myeloid suppressor cells. Given this finding, we tested whether the endogenous GM-CSF produced by LSK cells was sufficient for the generation of MDSCs. As expected, splenic LSK cells from Hepa mice generated highly suppressive myeloid descendants, without exogenous supplementation with GM-CSF, which could be completely prevented by the inclusion of anti-GM-CSF Abs in the culture (Figure 3, D and E). We consistently, obtained similar results using splenic LSK cells isolated from mice with DEN and $\mathrm{CCl}_{4}$-induced hepatoma and $A p c^{\mathrm{Min} /+}$ mice (Supplemental Figure 3, J and $\mathrm{K}$ ).

Given the distinct capability of splenic HSPCs to produce and respond to GM-CSF, we speculated that these primed progenitors could readily generate myeloid suppressors in vivo, even independently of the presence of tumors. To test this hypothesis, we transferred CD45.1 ${ }^{-}$splenic or BM LSK cells from Hepa mice into the spleens of naive $\mathrm{CD} 45.1^{+}$recipients. Five days after the adoptive transfer, we isolated donor cells and tested for their immunosuppressive activity (Figure 3F). Consistent with the in vitro findings discussed above, only the CD $45.1^{-} \mathrm{CD} 11 \mathrm{~b}^{+} \mathrm{Gr}-1^{+}$ myeloid descendant cells of splenic LSK cells, but not those derived from BM, could effectively inhibit anti-CD3- and antiCD28-stimulated proliferation and antigen-induced cytotoxic activity of T cells (Figure 3, G-I). These ex vivo data, together with the findings of endogenous GM-CSF expression and the effects of neutralizing $\mathrm{Ab}$, indicate that the splenic HSPCs in Hepa mice include primed progenitors of potent myeloid suppressors that can readily respond to the endogenous GM-CSF signal and support the suppressive myeloid response. 
A

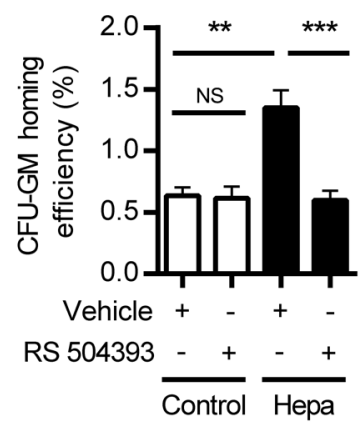

B

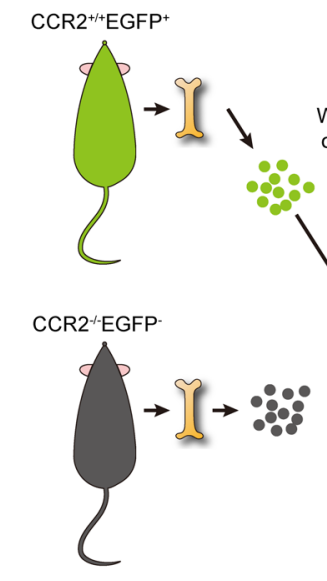

$\mathrm{CCR}^{+/+}(1$ and 2$)$

or CCR2 $2^{-1}$ (3 and 4$)$
E $\square$ Control and CCR2 ${ }^{+++} \square$ Control and CCR2 $\square$ Hepa and CCR2 ${ }^{+/+} \quad \square$ Hepa and CCR2 ${ }^{-1}$

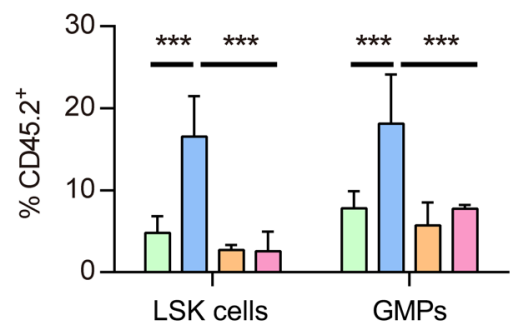

$\mathbf{F}$

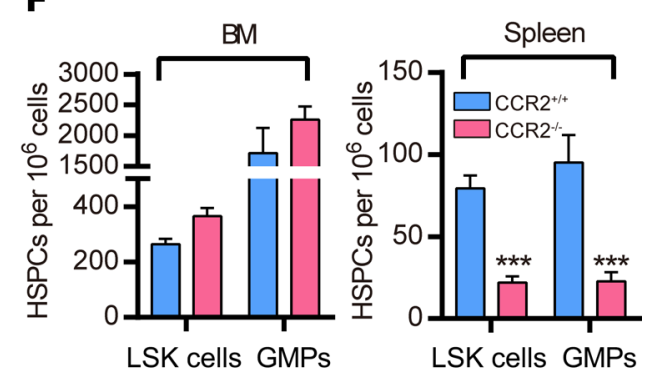

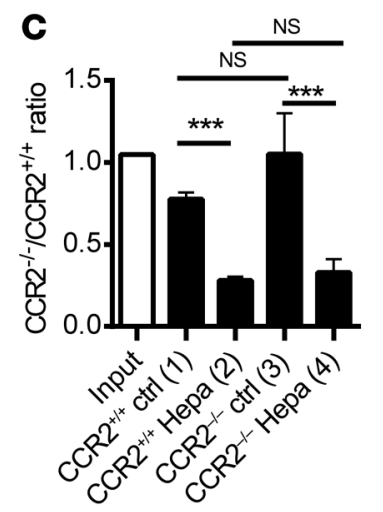

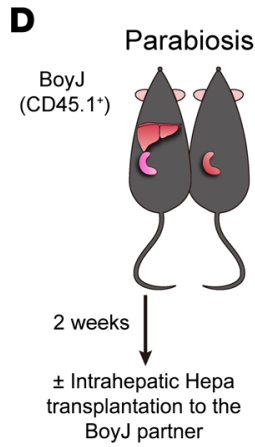

G

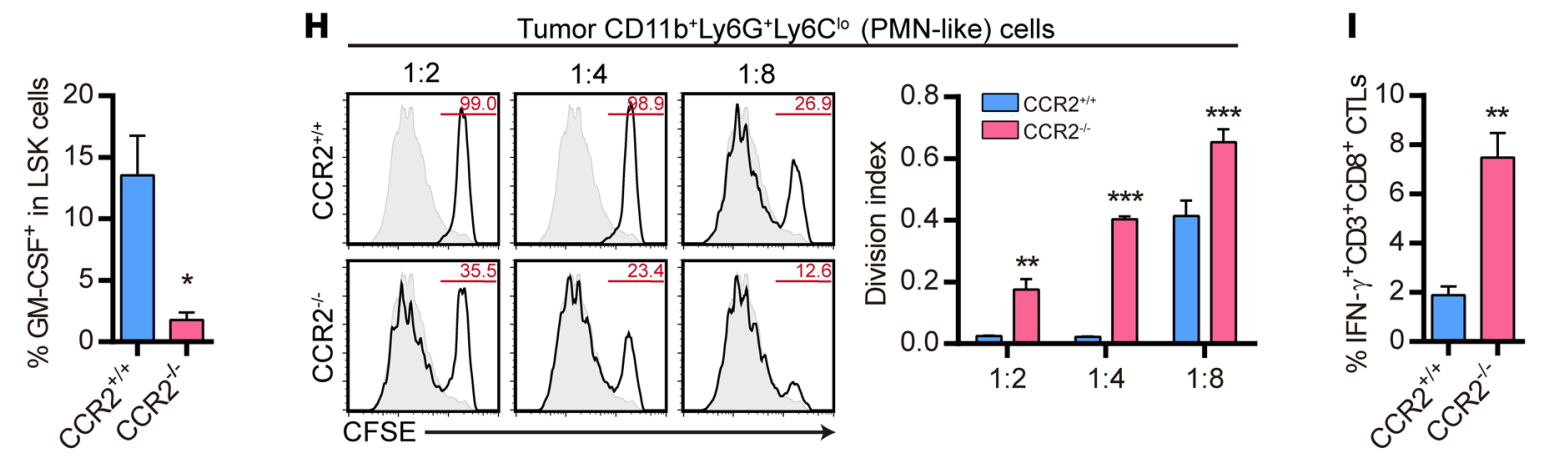

$\mathrm{CCR}^{+/+}$
or CCR2 (CD45.2+)

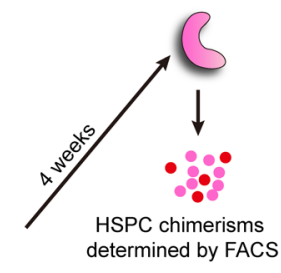

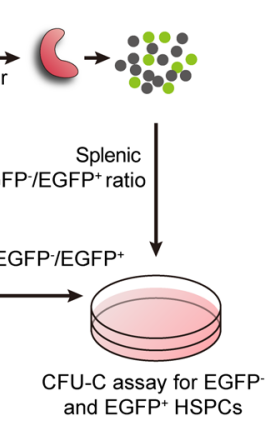

CFU-C assay for EGFP-

Figure 6. The CCL2/CCR2 axis mediates the enhanced splenic recruitment of circulating HSPCs in Hepa mice. (A) Percentages of transferred BM-derived CFU-GM progenitors homing to the spleens of control or Hepa mice. ${ }^{* *} P<0.01$ and ${ }^{* *} P<0.001$, by 1-way ANOVA followed by Tukey's test. (B) Scheme of competitive homing assays. (C) Ratios of ECFP-CCR2--/- versus ECFP+CCR2 ${ }^{+/+}$BM-derived CFU-CM progenitors homing to the spleens of control or Hepa mice ( $n$ $=8$ for CCR2 $^{+/+}$recipient groups; $n=4$ for CCR2 ${ }^{-/-}$recipient groups), as described in $\mathbf{B}$. ${ }^{* *} P<0.001$, by 1 -way ANOVA followed by Tukey's test. ctrl, control. (D) Cartoon depicting the generation of control and Hepa-bearing parabiotic pairs. (E) Flow cytometric quantification of CD45.2 ${ }^{+}$partner-derived splenic LSK and GMP cells from the parabiotic mouse pairs described in $\mathbf{D}$. ${ }^{* *} P<0.001$, by 2 -way ANOVA followed by Tukey's test. (F) Quantification of BM and splenic LSK and GMP cells in CCR2 $2^{++}$or CCR2 ${ }^{-/-}$Hepa mice. ${ }^{* *} P<0.001$, by Student's $t$ test. (G) GM-CSF expression in splenic LSK cells from CCR2 ${ }^{+/+}$or CCR2 ${ }^{-/-}$Hepa mice

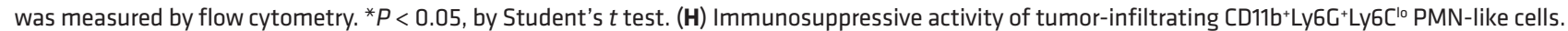
${ }^{* *} P<0.01$ and ${ }^{* * *} P<0.001$, by 2-way ANOVA with Bonferroni's correction. (I) Frequencies of tumor-infiltrating IFN- $\gamma^{+} \mathrm{CD} 3^{+} \mathrm{CD} 8^{+} \mathrm{CTLS}$ in $\mathrm{CCR2} 2^{+/+}$and $\mathrm{CCR2} 2^{-/-}$ Hepa mice. ${ }^{* *} P<0.01$, by Student's $t$ test. Data are representative of 2 experiments and presented as the mean \pm SEM. $n=3$ per group in each experiment (A-E); $n=4$ for the CCR2 ${ }^{+/+}$group, $n=5$ for the CCR2 $2^{-/-}$group in each experiment $(\mathbf{F}) ; n=3$ per group in each experiment $(\mathbf{G}-\mathbf{I})$.

Spleens of tumor-bearing mice recruit BM-derived circulating HSPCs. To investigate the underlying mechanism regulating the distinct properties of splenic LSK cells in Hepa mice, we first determined the cellular origin of these splenic HSPCs. The splenic HSPC population could be originated from the BM and/ or the clonal expansion of local residents. To determine the original source(s) of these accumulated HSPCs, we generated tumor-bearing parabiotic pairs by joining WT B6 and EGFP ${ }^{+}$ mice (Supplemental Figure 4A). After 4 weeks of parabiosis, the tumor dramatically induced partner-derived $\left(\mathrm{EGFP}^{+}\right)$HSPC accumulation in the spleens of B6 mice (Supplemental Figure $4, \mathrm{~B}$ and $\mathrm{C}$ ), suggesting circulating HSPCs compartment as an important source of splenic HSPCs. Importantly, removal of the spleen of the $\mathrm{EGFP}^{+}$partner barely altered the number, composition, or CFU in culture (CFU-C) activity of the partnerderived splenic HSPCs, excluding the possibility that a recircu- 

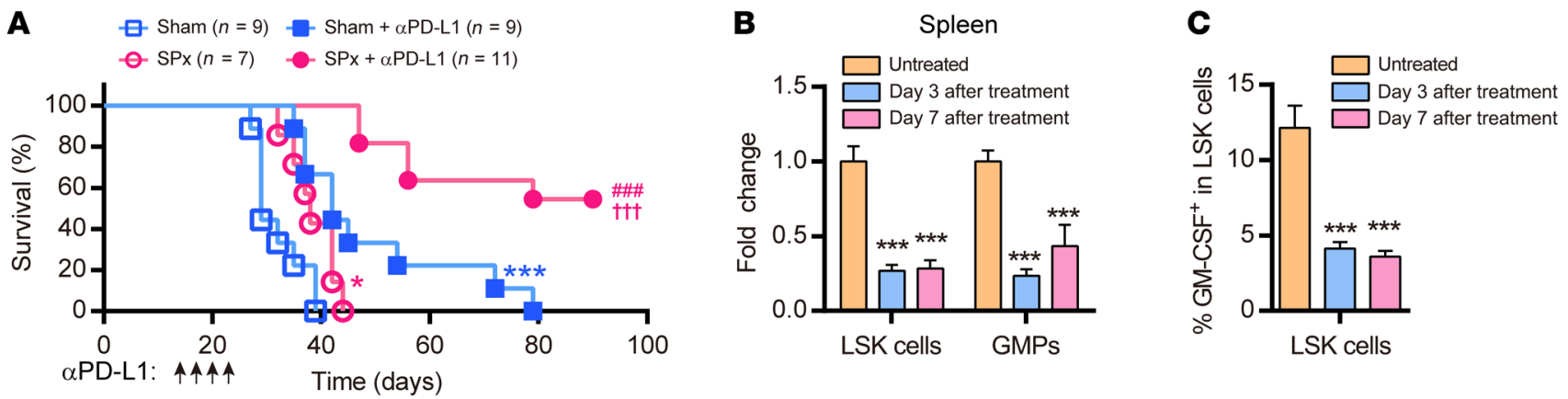

D

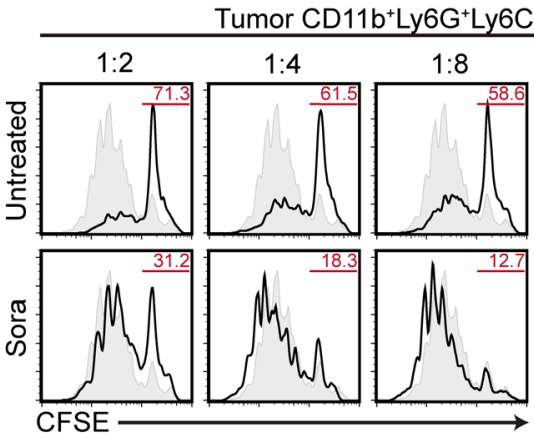

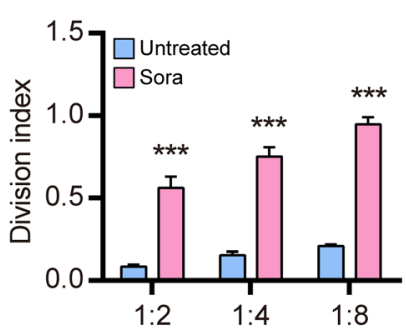

$\mathbf{E}$

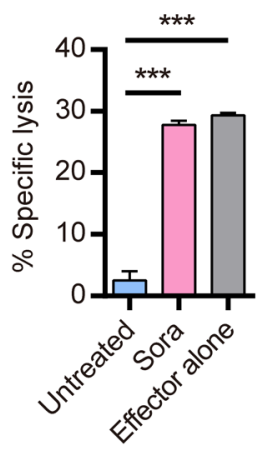

$\mathbf{F}$

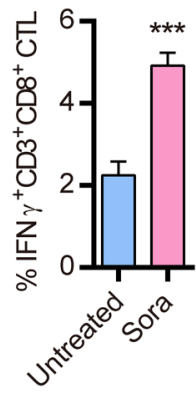

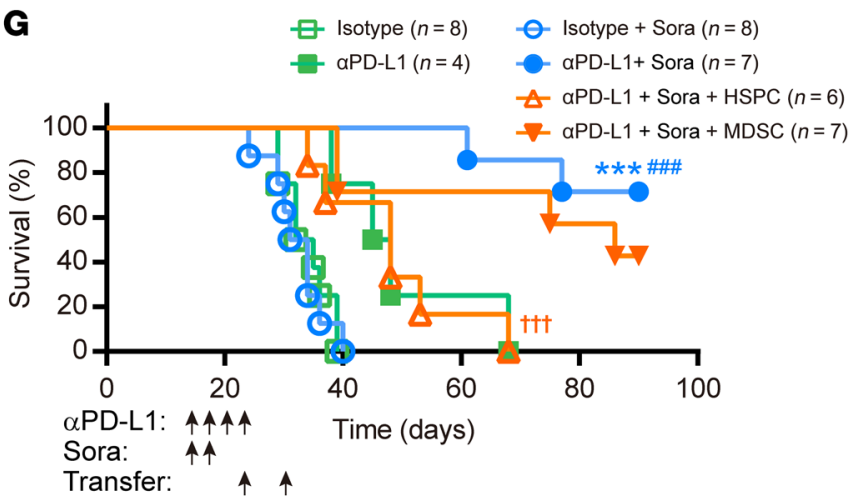

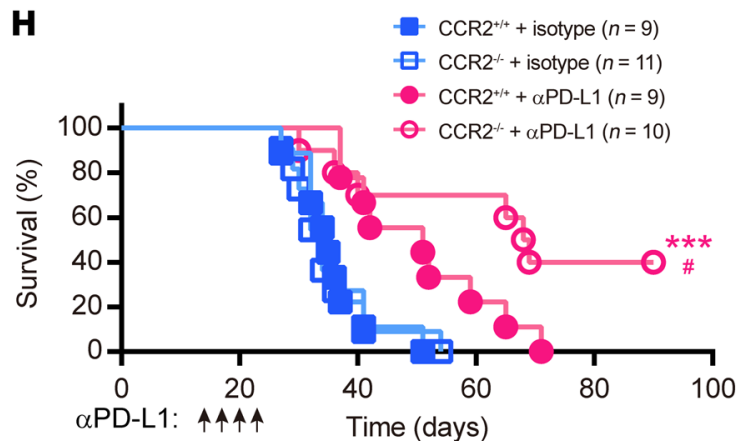

Figure 7. Abrogation of splenic EMH synergistically enhances anti-PD-L1 efficacy. (A) Survival of Hepa mice subjected to sham surgery or splenectomy with or without anti-PD-L1 treatment. ${ }^{*} P<0.001$ and ${ }^{* * *} P<0.001$, by log-rank test compared with the sham group; ${ }^{\# \# \# ~} P<0.001$, by log-rank test compared with the splenectomy group; ${ }^{\dagger+t} P<0.001$, by log-rank test compared with the sham plus anti-PD-L1 group. (B) Fold changes in the numbers of splenic LSK and GMP cells in Hepa mice. ${ }^{* * *} P<0.001$, by 2-way ANOVA followed by Dunnett's test. (C) Proportions of GM-CSF ${ }^{+}$splenic LSK cells in Hepa mice. ${ }^{* *} P<0.001$, by 2-way ANOVA followed by Dunnett's test. (D-F) Immunosuppressive activity of tumor-infiltrating CD11b ${ }^{+} L y 6 C^{+}+{ }^{*} 6 C^{10} P M N-l i k e$ cells ( $\mathbf{D}$ and $\mathbf{E}$ ) and the frequencies of tumor-infiltrating IFN- $\gamma^{+} \mathrm{CD} 3^{+} \mathrm{CD} 8^{+} \mathrm{CTLs}(\mathbf{F})$. Hepa mice were treated with low-dose sorafenib (Sora) 7 days prior to examination or were left untreated as controls. ${ }^{* *} P<0.001$, by 2 -way ANOVA with Bonferroni's correction. (G) Survival of Hepa mice that received no treatment or treatment with low-dose sorafenib or anti-PD-L1 or both. For some groups, mice received adoptive transfers of $1 \times 10^{6}$ splenic HSPCs or $5 \times$ $10^{6}$ MDSCs 3 and 10 days after the latter dose of sorafenib. ${ }^{* * *} P<0.001$, by log-rank test compared with the isotype plus sorafenib group; \#\#\# $P<0.001$, by log-rank test compared with the anti-PD-L1 group; ${ }^{\dagger+} P<0.001$, by log-rank test compared with the anti-PD-L1 plus sorafenib group. (H) Survival of CCR2 ${ }^{+/+}$ or CCR2 ${ }^{-1-}$ Hepa mice treated with anti-PD-L1 or isotype Abs. ${ }^{* *} P<0.001$, by log-rank test compared with the CCR2 ${ }^{-1-}$ plus isotype group; ${ }^{*} P<0.05$, by log-rank test compared with the CCR2 ${ }^{+/+}$plus anti-PD-L1 group. Data are representative of 2 experiments (B-F; $n=3$ per group in each experiment) and indicate the mean \pm SEM, or were pooled from 2 experiments ( $n=7-11$ per group as indicated in $\mathbf{A}, \mathbf{G}$, and $\mathbf{H}$ ).

lating splenic resident cell population was the major source. In addition, the spleens of tumor-bearing mice showed a 2 -fold greater capacity for recruiting circulating BM-derived CFUGM progenitors (Supplemental Figure 4, D and E). Thus, these results suggested that the majority of splenic HSPCs in Hepa mice were derived from the circulating cell population that originated from BM.
The splenic niche modulates LSK HSPC functional properties. Since the majority of splenic HSPCs are BM derived, we sought to investigate the mechanism(s) accounting for their distinct functions compared with those of their counterparts in BM. We reasoned that this difference could possibly result from the local "education" by the cancer-conditioned splenic niche and/or the selective recruitment of circulating HSPCs. 

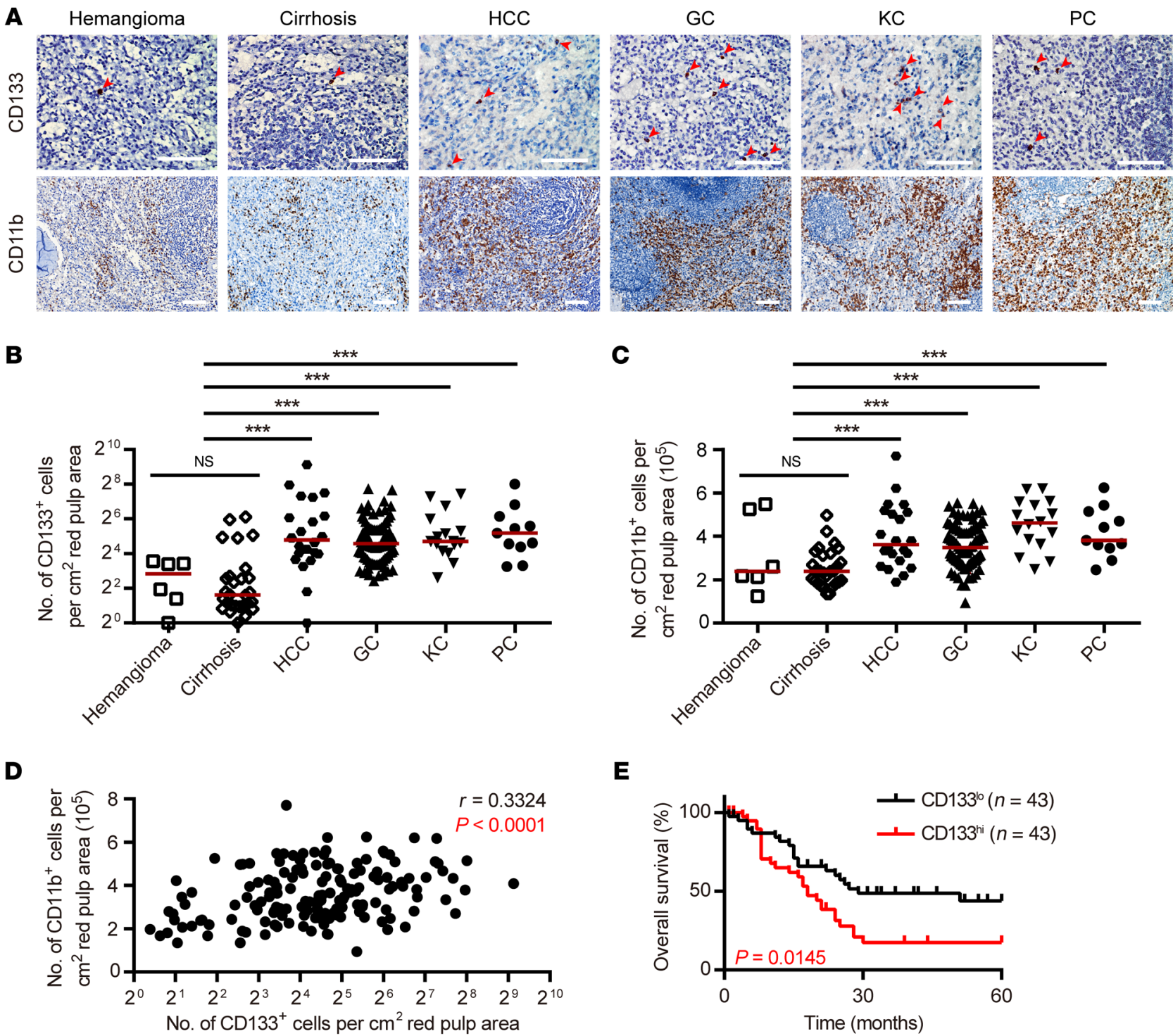

Figure 8. Splenic EMH in patients with different types of solid tumors. (A) Representative immunohistochemical staining of CD133 ${ }^{+}$and CD11 $\mathrm{b}^{+}$cells in splenic specimens from a total of 135 patients with hepatocellular carcinoma (HCC; $n=22$ ), gastric cancer (GC; $n=86$ ), kidney cancer (KC; $n=16$ ), or pancreatic cancer (PC; $n=11)$ and from 34 noncancer patients with splenic hemangioma $(n=6)$ or cirrhosis $(n=28)$. Red arrows point to the CD133+ cells in the fields. Scale bars: $50 \mu \mathrm{m}$. (B and C) Quantification of CD133+ HSPCs (B) and CD11b myeloid cells (C) in the splenic specimens described in $\mathbf{A} .{ }^{* * *} P<0.001$, by Kruskal-Wallis test. (D) Correlation between splenic CD133+ HSPC and splenic CD11b+ myeloid cell densities. The results of a Spearman's rank correlation analysis are indicated. (E) Patients with gastric cancer were divided into 2 groups according to the median value of the splenic CD133+ cell frequencies. The duration of overall survival was estimated using the Kaplan-Meier method and compared by log-rank test $(P=0.0145)$.

We first tested the "local education" hypothesis by coculturing naive BM LSK HSPCs with BM or splenic stromal cells isolated from control or Hepa mice (Figure 4A). The results showed that only the splenic stromal cells from Hepa mice could induce an increase in the CFU-GM activity (Figure 4B) and endogenous GM-CSF expression (Figure 4C) of BM-derived naive LSK cells. To determine whether splenic stromal cells could alter the capability of these naive early HSPCs in vivo, we adoptively transferred naive BM LSK cells into the spleens of control or Hepa mice (Figure 4D). We observed that splenic stroma of Hepa mice, but not that of control mice, induced these early progenitors to produce highly suppressive myeloid descendants in vivo (Figure $4 \mathrm{E}$ ). These data indicate that the cancer-conditioned splenic niche is capable of educating naive LSK cells to generate myeloid immune suppressors.

This education effect was retained in the Transwell culture system (Figure 4, F-H), suggesting that this effect was mediated by soluble factor(s). We found that IL-6 was upregulated in the splenic stromal cells of Hepa mice (Supplemental Figure 4, F and G). The inclusion of anti-IL-6-neutralizing Abs in the Transwell assays attenuated the CFU-GM activity (Figure 4F) and GM-CSF expression (Figure 4G) of LSK cells cultured with Hepa splenic stromal cells and impaired the suppressive function of their myeloid descendants (Figure $4 \mathrm{H}$ ). In support of this, recombinant mouse IL-6 induced GM-CSF expression in LSK cells (Supple- 
mental Figure $4 \mathrm{H}$ ). Together, these data demonstrate that splenic stromal cells of tumor-bearing mice can induce LSK cell functional alteration via soluble factors such as IL-6.

Upregulation of CCL2/CCR2 signaling in the spleen. Having revealed the role of the local education, we next investigated whether a selective recruitment mechanism might also contribute to this splenic HSPC response. Interestingly, we observed higher chimerisms in both splenic LSK and LK cells in Hepa tumor-bearing parabiotic pairs (Supplemental Figure 5A), suggesting a faster turnover of splenic HSPCs, which contradicted their accumulation. To further define the kinetics of HSPC turnover in the spleen, we surgically separated pairs of mice after 4 weeks of parabiosis. After separation, splenic HSPC chimerisms in tumor-free pairs were unchanged during the 96-hour observation, whereas the chimerisms in tumor-bearing mice significantly decreased (Supplemental Figure 5B). These results indicate a cancer-driven increase in splenic HSPC turnover, possibly due to their faster differentiation, since the apoptotic rates of splenic HSPCs were similar between the control and Hepa mice (Supplemental Figure 5, C-E). Thus, we reasoned that the accumulation of splenic HSPCs in Hepa mice might rely on the cancer-endowed stronger attraction for BM-derived circulating progenitor cells (Supplemental Figure $4 \mathrm{E}$ ), thus outstripping the accelerated HSPC consumption.

To probe the mechanism involved in the recruitment of circulating HSPCs into the spleen, we examined the expression profiles of cytokines and chemokines (Supplemental Figure 6A). The CXCR4 ligand CXCL12 (also known as stromal cell-derived factor 1) is not only a critical mediator for HSPC recruitment and retention in BM, but also mediates splenic EMH induced by myeloablation, blood loss, or pregnancy (33). We were surprised to find that CXCL12 was downregulated in the spleens of tumor-bearing mice (Figure 5A and Supplemental Figure 6B). In contrast, the CCR2 ligand CCL2, along with the CXCR2 ligands CXCL2 and CXCL5, was among the most upregulated chemokines in the spleens of Hepa mice (Figure 5B and Supplemental Figure 6C). Since CXCR2 was not expressed on BM or splenic HSPCs (Supplemental Figure 6D), we focused on the CCL2/CCR2 axis in our study. To determine the potential source(s) of splenic CCL2, we analyzed its production in different splenic cell populations. Splenic stromal cells, especially VE-cadherin ${ }^{+}$endothelial cells, markedly increased CCL2 production in Hepa mice (Figure 5C and Supplemental Figure 6E). We then analyzed the expression of the corresponding chemokine receptor CCR2 on HSPCs and found that a remarkably larger proportion of splenic LSK HSPCs from Hepa mice expressed CCR2 compared with those from BM or control spleen (Figure 5D).

Role of the CCL2/CCR2 axis in the splenic recruitment of myeloid HSPCs. To investigate the role of the CCL2/CCR2 axis in mediating splenic recruitment of circulating myeloid progenitors, we measured CFU-GM homing. RS 504393, a highly selective CCR2 antagonist, effectively reduced the splenic recruitment of circulating CFU-GM progenitors in tumor-bearing mice to basal levels (Figure 6A). To corroborate the direct action of CCL2 on HSPCs, we transferred CCR2-deficient (EGFP ${ }^{-}$) and CCR2-competent $\left(\mathrm{EGFP}^{+}\right) \mathrm{BM}$ cells simultaneously into control (groups 1 and 3) or tumor-bearing (groups 2 and 4) recipients (Figure 6B). Irrespective of the CCR2 expression status in recipi- ent mice, the ratios of $\mathrm{CCR}^{-/-} \mathrm{EGFP}^{-}$to $\mathrm{CCR} 2^{+/+} \mathrm{EGFP}^{+} \mathrm{CFU}-\mathrm{GM}$ progenitors homing to the spleens of tumor-bearing hosts were at least 3-fold lower compared with the ratios of input and those in control spleens (Figure 6C).

Next, we tracked CCR2-deficient and CCR2-competent HSPCs in parabiotic pairs with or without Hepa tumors (Figure 6D). The proportion of CCR2-competent, partner-derived (CD45.2+) splenic HSPCs was significantly increased in the tumor-bearing pairs. As expected, this substantial elevation in the partner-derived HSPC proportion was abrogated if partner-derived circulating progenitor cells lacked CCR2 expression (Figure 6E). Likewise, the tumor-elicited splenic accumulation of LSK and GMP cells was attenuated in $\mathrm{CCR} 2^{-/}$Hepa mice, while we observed little change in the BM (Figure 6F). These results indicate that CCL2-CCR2 interaction directly mediates the splenic recruitment of circulating HSPCs with myeloid potential in tumor-bearing hosts. More important, the remaining splenic LSK cells in CCR2-deficient Hepa mice had markedly decreased levels of endogenous GM-CSF expression (Figure 6G), suggesting that the education by the spleen required the peripheral CCR2 ${ }^{+}$LSK subset (34).

Accordingly, lack of CCR2 expression on HSPCs recapitulated the effect of splenectomy on the tumor-promoting myeloid response. The absence of the endogenous GM-CSF-driven splenic EMH impaired the suppressive activity of tumor PMN-like cells in CCR2-deficient Hepa mice (Figure 6H and Supplemental Figure $6 \mathrm{~F}$ ), which allowed an increase in the number of tumor-infiltrating IFN $-\gamma^{+} \mathrm{CD}^{+} \mathrm{CD}^{+}$CTLs (Figure 6I). Although knockout of CCR2

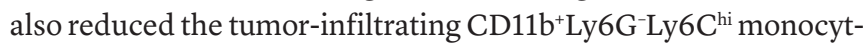
ic cells (Supplemental Figure 6G), this was supposed to be a minor effect compared with the functional alteration of PMN-like cells, given the relatively smaller number and less suppressive ability of these cells in the Hepa model (Supplemental Figure 3, A-D). Thus, these findings indicate that the recruitment of HSPCs via the CCL2/CCR2 axis, followed by the splenic education, is an integral and essential process for inducing the distinct splenic EMH that promotes the systemic myeloid response.

Abrogation of splenic EMH synergistically enhances anti-PD-L1 therapy. Given the above findings that the abrogation of splenic EMH enhanced antitumor immunity (Figure 3), we expected that it might potentially promote immunotherapy. Hepa mice were treated with splenectomy or sham surgery and sacrificed when the tumor reached a diameter of approximately $1.5 \mathrm{~cm}$. In Hepa mice inoculated with $7.5 \times 10^{5}$ tumor cells, the impact of splenectomy on mouse survival was mild but statistically significant (Figure 7A). The effect of splenectomy was more robust when the initiating tumor cells were reduced (Supplemental Figure 7A). This agrees with previous reports suggesting that the impact of splenectomy on cancer progression is dependent on the tumor settings in animal models and is conditional in patients $(21,35-37)$. We noticed that splenectomy did not decrease the expression of PD-L1 on tumor PMN-like cells (Supplemental Figure 3F), which might indicate an opportunity for combined therapy with anti-PD-L1 $(38,39)$. Monotherapy of anti-PD-L1 treatment did not affect the number or phenotype of splenic HSPCs (Supplemental Figure 7, B-D) and only elicited marginal therapeutic effects in Hepa mice (Figure 7A) and in another hepatoma mouse model based on a $\mathrm{BALB} / \mathrm{c}$ background (Supplemental Figure 7, E and F). However, 
splenectomy synergistically enhanced the therapeutic efficacy of anti-PD-L1 treatment (Figure 7A and Supplemental Figure 7F). Complete regression of established cancer was achieved in over $50 \%$ of Hepa mice. These results suggest a potential role for targeting splenic EMH to enhance immunotherapy.

In order to preserve the normal physiological functions of the spleen while inhibiting splenic EMH, we tested whether selectively targeting splenic HSPCs could also synergize with immunotherapies. Low-dose sorafenib, a c-Kit inhibitor (40), induced the apoptosis of splenic HSPCs (Supplemental Figure 7G), inhibited their proliferation (Supplemental Figure $7 \mathrm{H}$ ), and reduced the numbers of HSPCs in spleen but had much less effect on those in the BM (Figure 7B and Supplemental Figure 7I). Moreover, low-dose sorafenib treatment attenuated endogenous GM-CSF expression in splenic LSK cells (Figure 7C). Consistent with splenectomy, low-dose sorafenib inhibited the suppressive effects of tumor PMN-MDSCs on T cell proliferation and cytotoxic activity (Figure 7, D and E), increased the frequencies of infiltrating IFN- $\gamma^{+}$ $\mathrm{CD}^{+} \mathrm{CD}^{+}$CTLs (Figure 7F), and recapitulated the synergistic effect when combined with PD-L1 blockade (Figure 7G). Importantly, as shown in Figure 7G, this synergistic effect was abrogated by restoring the splenic $\mathrm{Lin}^{\mathrm{lo} /}{ }^{-} \mathrm{c}-\mathrm{Kit}^{\mathrm{hi}}$ population including LSK and downstream myeloid progenitor cells, which are capable of generating myeloid suppressor cells (Supplemental Figure 3, H and I), but not by transferring splenic MDSCs, possibly because of their short lifespan (11). These data demonstrate the important role of splenic EMH in impeding antitumor immunity and immunotherapy. Likewise, anti-PD-L1 treatment elicited profound therapeutic responses in CCR2-deficient Hepa mice (Figure 7H). Together, these findings demonstrate that targeted therapies against splenic EMH can effectively synergize with immune checkpoint blockade and thus may represent a novel strategy to enhance currently available immunotherapy.

Splenic EMH in patients with different types of solid tumors. Having established the function, mechanism, and significance of tumor-induced splenic EMH in mouse models, we sought to assess the clinical relevance of splenic EMH. CD133 is a marker for human HSPCs (41). We confirmed that splenic CD133+ cells had a Lin $^{-}$CD34 ${ }^{\text {hi }}$ phenotype (Supplemental Figure 8A) and were highly enriched for CFU-GM activity (Supplemental Figure 8B). We examined the frequencies of splenic HSPCs in situ by staining for CD133 in formalin-fixed, paraffin-embedded splenic tissues from 135 patients with different types of solid tumors and 34 noncancer patients (Supplemental Table 1). The frequencies of splenic $\mathrm{CD}_{133^{+}}$cells in the patients with cancer were significantly higher than those in patients without cancer (Figure 8, A and B, and Supplemental Figure 8C). The densities of CD11 ${ }^{+}$myeloid cells were also markedly increased in the spleens of cancer patients (Figure 8, A and C), which significantly correlated with the splenic CD133+ HSPC levels (Figure 8D).

Finally, we evaluated the clinical significance of cancerinduced splenic accumulation of HSPCs. Kaplan-Meier analyses revealed that the high frequency of $\mathrm{CD} 133^{+}$progenitors in the spleen negatively impacted the overall survival of patients with gastric cancer $(P=0.0145$, hazard ratio $[\mathrm{HR}]=2.096,95 \% \mathrm{CI}$ : 1.158-3.794) (Figure 8E). Patients with lower splenic CD133+ cell frequencies survived significantly longer (median, 29 months) than did patients with higher splenic $\mathrm{CD} 133^{+}$cell frequencies (median, 18 months). This analysis of patients with other types of tumors was not applicable yet, either because of an insufficient sample size or because the majority of the patients were recently diagnosed at the early stage (Supplemental Table 1). Overall, in line with our findings in mouse models, the clinical data presented so far suggest the functional and clinical importance of splenic $\mathrm{EMH}$ in patients with cancer.

\section{Discussion}

Cancer is associated with deviations of host hematopoiesis in both $\mathrm{BM}$ and extramedullary niches $(20,21,42)$. The present study indicates that the spleen, as a major site of cancer-induced $\mathrm{EMH}$, actively recruits HSPCs via the CCL2/CCR2 signaling pathway and supports their endogenous GM-CSF-driven differentiation into potent myeloid suppressor cells. These findings demonstrate that the spleen could serve as a distinct niche for priming myeloid HSPCs to fuel the tumor-promoting myeloid response.

Extramedullary niches of hematopoiesis can be established to exert critical influence to alleviate $(43,44)$ or deteriorate $(34$, 45-48) pathological conditions. However, our knowledge about the nature and properties of extramedullary HSPCs is very limited, and the necessity and advantages of outsourcing hematopoiesis, especially under chronic pathological circumstances, remain unclear. In cancer, splenic EMH is generally perceived as a supplementary mechanism serving to fulfill the increased demand of myeloid cells (22). Thus far, the relative contribution of splenic $\mathrm{EMH}$, compared with BM myelopoiesis, to the systemic tumor-associated myeloid response seems dependent on model set-ups. This EMH has been attributed to the generation of the majority of tumor-associated macrophages in a genetic mouse model of lung adenocarcinoma $(20,21,49)$, while it represents a quantitatively minor source of tumor-infiltrating myeloid cells in some other models $(42,50)$. Apart from the comparison of production capacity, we provide evidence here that cancer-induced splenic myelopoiesis functionally and mechanistically differs from its BM counterpart and plays a significant role in the tumor-promoting myeloid response. In tumor-bearing mice, splenic LSK HSPCs, selectively recruited via the CCL2/CCR2 axis, are educated by the splenic niche to express higher levels of GM-CSF, acquire heightened proliferative activity, and become committed to generating potent immunosuppressive myeloid cells. Therapeutically, targeted abrogation of splenic EMH alone (without affecting the $\mathrm{BM}$ ) is sufficient to synergistically enhance the therapeutic efficacy of immune checkpoint blockade, suggesting an important role for this EMH in tipping the balance of tumor immune responses. Taken together, our findings provide insights into the functional specialty of splenic hematopoiesis in cancer.

HSPCs are not only able to sense stress signals but are also capable of converting environmental cues into versatile cytokine signals to regulate hematopoiesis in an autocrine/paracrine manner (30). The present study reveals a positive feedback mechanism of endogenous GM-CSF in splenic LSK HSPCs that primes their myeloid differentiation and fully exploits their profound potential for generating myeloid suppressor cells. The regulation of HSPC activity and MDSC generation has long been attributed to systemic signals derived from tumors $(12,18-20,31,32)$. Complementary 
to these findings, the present study suggests an alternative mode of regulation, in which one crucial endogenous signal from primed progenitor cells could be required and sufficient. However, this priming process is tightly regulated, as recruitment of circulating HSPCs via the CCL2/CCR2 axis and niche signals provided by the splenic stromal cells are both essential preconditions for eliciting such a HSPC response. These findings may help to elucidate the mechanisms regulating divergent HSPC activities at different sites of hematopoiesis.

An effective and durable antitumor immune response requires an intact "cancer immunity cycle," which allows a series of immune events to initiate, proceed, and expand iteratively (51). In some patients, monotherapeutic approaches are sufficient to restore and reinitiate such a self-sustaining cycle of cancer immunity, whereas others may require combined therapies $(38,39,52-$ $54)$. Although the impact of splenectomy on cancer progression has been shown to be generally limited in the clinic $(35,37)$, the beneficial effect is more robust in a subset of patients with higher levels of myelopoiesis (36). In combination with immunotherapies such as adoptive transfer of tumor antigen-specific CTLs (42) or immune checkpoint blockade (the present study), the abrogation of splenic myelopoiesis shows promising effects in enhancing therapeutic antitumor immune responses. Moreover, targeted abrogation of splenic EMH while preserving other physiological functions of the spleen, e.g. by low-dose c-Kit inhibitor or blockade of CCL2/CCR2 signaling, can be equally effective and may be more appropriate for clinical practice. These findings indicate the important role of splenic EMH in tumor immunity, suggesting that selective abrogation of this process may create conditions that potentiate anticancer immunotherapy.

Although splenic EMH in patients with cancer has been suggested to be analogous to that in tumor-bearing mice (21), its clinical relevance remains unclear $(22,35,37)$, largely because of sample restrictions and technical limitations. The present study demonstrates that CD133 could serve as a useful marker for splenic HSPCs in humans. Using this marker, we have unveiled the general existence and clinical relevance of cancer-associated splenic EMH in patients. Importantly, higher splenic CD133+ cell frequencies are associated with poorer patient prognosis, at least in gastric cancer. Still, further investigation into the relationship between splenic EMH and the immunosuppressive systemic (macro-) and tumor (micro-) environment is warranted. In addition, clinical data from larger patient cohorts and more types of cancers are required to further address the exact extent of involvement of splenic EMH in tumor immunity, which will be an area of significant interest for future studies.

In summary, our work describes what to our knowledge are previously unrecognized features of cancer-induced splenic EMH and indicates its unique and important role in both mouse models and cancer patients. Our findings propose a 2-step model for the distinct cancer-induced splenic HSPC response: (a) the CCR2-dependent recruitment of HSPCs, followed by (b) the local induction of endogenous GM-CSF signaling, which drives HSPC myeloid commitment and differentiation into potent immunosuppressive myeloid cells. Identifying these unique signals and mechanisms that instruct splenic EMH may provide a novel strategy for cancer therapy, by targeting the tumor-promoting myeloid response at its source.

\section{Methods}

Animals. All mice were maintained under specific pathogen-free conditions in the animal facilities of Sun Yat-sen University Cancer Center. Details on the mice are provided in the Supplemental Methods.

Tumor models and treatments. An orthotopic hepatic tumor was established via subcapsular intrahepatic injection of $7.5 \times 10^{5}$ Hepa16 tumor cells (except for the experiment described in Supplemental Figure 7A), suspended in $25 \mu \mathrm{l}$ of $50 \%$ Basement Membrane Extract (Trevigen), into the left lobe of the livers of anesthetized 6- to 8-week-old B6 mice. The mice were sacrificed when the tumor reached a diameter of approximately $1.5 \mathrm{~cm}$ or showed any signs of agony. Details on the other mouse models used in this study are provided in the Supplemental Methods. For therapeutic anti-PD-L1 treatment, $200 \mu \mathrm{g}$ anti-PD-L1 (clone 10F.9G2, Bio X Cell) or the corresponding IgG2b isotype control (Bio X Cell) in $100 \mu \mathrm{l}$ PBS was administered intraperitoneally into mice 4 times at 3-day intervals, 2 weeks after tumor cell transplantation. For sorafenib treatment, the mice received 2 doses of $20 \mathrm{mg} / \mathrm{kg}$ sorafenib (Nexavar, Bayer) or vehicle $(100 \mu$ sterile water) by oral gavage at an interval of 3 days. For CCR2 antagonist treatment, mice received 2 mg/kg RS 504393 (MilliporeSigma) or vehicle (1\% DMSO in PBS) by oral gavage 3 hours before the adoptive cell transfer.

Patients. Splenic samples were obtained from patients who underwent a splenectomy at the Sun Yat-sen University Cancer Center or The Third Affiliated Hospital of Sun Yat-sen University. None of the patients had received anticancer therapy prior to the sampling, and no splenic metastasis was observed. Individuals with concurrent autoimmune disease, HIV, or syphilis were excluded. Clinical stages were classified according to the guidelines of the International Union Against Cancer. Details on patient information are provided in Supplemental Table 1 and the Supplemental Methods.

Flow cytometry. Flow cytometry was performed as previously described $(55,56)$. Details of the Abs used for flow cytometry are listed in Supplemental Table 2. The procedures for staining are described in the Supplemental Methods. Data were acquired on a Gallios or a Cytoflex S flow cytometer (Beckman Coulter) and analyzed with Kaluza Analysis (Beckman Coulter) and FlowJo Software (Tree Star).

$\mathrm{CFU}$ assays. CFU- $\mathrm{S}_{12}$ assays were performed as previously described $(57,58)$. Briefly, isolated LSK cells from the BM or spleen were intravenously transferred into lethally irradiated recipient mice. After 12 days, the spleens of the recipient mice were fixed in Bouin's solution (MilliporeSigma), and the colonies were counted. CFU-C assays were carried out using complete methylcellulose-based medium (MethoCult GF M3434 or H4034, STEMCELL Technologies) following the manufacturer's instructions. In some experiments, anti-mouse GM-CSF ( $3 \mu \mathrm{g} / \mathrm{ml}$; eBioscience) or the corresponding isotype control $\mathrm{Ab}$ was added to the medium. Colony numbers and morphology were assessed after 12 to 14 days of culture. For quantification, BM CFU activity was estimated by calculating the colonies in the BM of 1 femur and 1 tibia per mouse. A 2-step culture assay was also performed to examine the multilineage differentiation capability of single HSPCs. Single LSKs or GMPs were deposited into 96-well plates containing $100 \mu \mathrm{l}$ Serum-Free Expansion Medium (StemSpan SFEM, Stem Cell Technologies) supplemented with SCF $(50 \mathrm{ng} / \mathrm{ml})$ and TPO (10 ng/ml). After 4.5 days of culture, single-cell-derived daughter cells were transferred into methylcellulose-based medium (MethoCult GF M3434, containing SCF, erythropoietin [EPO], IL-3, 
and IL-6) supplemented with thrombopoietin (TPO) $(10 \mathrm{ng} / \mathrm{ml})$. Secondary colonies were counted after 10 days. All cultures were incubated at $37^{\circ} \mathrm{C}$ in a $5 \% \mathrm{CO}_{2}$ humidified atmosphere.

IHC. Paraffin-embedded samples were cut into 3- to $4-\mu \mathrm{m}$ sections and processed for IHC as previously described $(55,56)$ with minor modifications. The detailed procedures are provided in the Supplemental Methods. Slides were imaged using the Vectra Intelligent Analysis System (PerkinElmer). Quantification of positive signals and evaluation of splenic red and white pulp areas were performed with InForm 2.1 (PerkinElmer). For human $\mathrm{CD}_{133^{+}}$cell quantification, evaluation of $\mathrm{CD} 133^{+}$cells was performed by 2 independent observers who were blinded to the diagnosis and clinical outcome.

HSPC culture. HSPCs were isolated by FACS and cultured in Serum-Free Expansion Medium (StemSpan SFEM, STEMCELL Technologies) supplemented with the indicated recombinant cytokines and/or Abs as described in Figure 1D; Figure 2, D and E; Figure 3, D and E; Figure 4H, Supplemental Figure 1I, and Supplemental Figure $3 \mathrm{H}, 3 \mathrm{~J}$ and $3 \mathrm{~K}$. Cells were cultured at $37^{\circ} \mathrm{C}$ in a $5 \% \mathrm{CO}_{2}$ humidified atmosphere. Details on cell cultures are provided in the Supplemental Methods.

$T$ cell proliferation assay. Naive splenocytes were labeled with $2 \mu \mathrm{M}$ CFSE (Molecular Probes) and cultured in RPMI 1640 supplemented with 10\% FBS, $20 \mathrm{U} / \mathrm{ml}$ recombinant IL-2 (eBioscience), $1 \mu \mathrm{g} / \mathrm{ml}$ anti-CD3 (eBioscience), and $5 \mu \mathrm{g} / \mathrm{ml}$ anti-CD28 (eBioscience). Freshly isolated myeloid cells or $\mathrm{Gr}-1^{+}$cells derived from cultured HSPCs were incubated with the splenocytes at the ratio of 1:4, unless otherwise indicated (Figure 3, A and H; Figure 4E; Figure 6H; Figure 7D; and Supplemental Figure 3, C, H, and I). After 72 hours of coculture, CFSE dilution was assessed, and splenocyte proliferation was analyzed. Within each experiment, all test groups were internally controlled and compared with the splenocyte-alone control group (shown as shaded areas in the histograms). The division index, which is the average number of cell divisions that a cell in the original population has undergone, was calculated using FlowJo and normalized to the splenocyte-alone group in each experiment.

$T$ cell cytotoxicity assay. The CTL assay we performed was based on a mixed leukocyte peptide culture as previously described $(17,59)$. The culture was set up with $6 \times 10^{5}$ irradiated B6 splenocytes pulsed with $1 \mu \mathrm{g} / \mathrm{ml} \mathrm{OVA}_{257-264}$ peptide as feeder cells and incubated together with $1.2 \times 10^{4}$ OVA-specific CD $8^{+} \mathrm{T}$ effector cells isolated from the spleens of OT-I mice. Freshly isolated myeloid cells or Gr-1 ${ }^{+}$cells $(2.5 \times$ $10^{4}$ ) derived from cultured HSPCs were added as a third component in the culture, except for the effector alone control group. After 5 days, the cultures were tested for the ability to lyse the $\mathrm{OVA}_{257-264}$ peptide-pulsed Hepa 1-6 cells in a nonradioactive cytotoxicity assay measuring lactate dehydrogenase release (Promega). The percentage of specific lysis was calculated from triplicate samples as follows: (experimental $\mathrm{OD}_{490}$ spontaneous $\left.\mathrm{OD}_{490}\right) /\left(\right.$ maximal $\mathrm{OD}_{490}$ - spontaneous $\left.\mathrm{OD}_{490}\right) \times 100 \%$.
Homing assays. BM cells from donor mice were intravenously transferred into lethally irradiated recipient mice. After 16 hours, the splenocytes of recipient mice were harvested. Homing efficiency was evaluated by calculating the percentage of injected CFU-GM that was retrieved in the recipients' spleens.

Statistics. All statistical tests were 2 sided. For normally distributed data, we applied the Student's $t$ test, and the nonparametric exact Wilcoxon signed-rank test was used to compare data that were not normally distributed. For multiple comparisons (including multiple 2-group comparisons shown in the same panel), a 1-way or 2-way ANOVA (for parametric data) followed by the Bonferroni's correction (only 2 groups were compared), the Dunnett's test (all groups were compared with 1 control group), or the Tukey's multiple comparisons test (all groups were compared with each other). For nonparametric data, a Kruskal-Wallis test, followed by Dunn's multiple comparisons test, was applied. Cumulative survival time was estimated using the Kaplan-Meier method, and the log-rank test was applied to compare the groups. $P$ values of less than 0.05 were considered statistically significant.

Study approval. All animal experiments were performed according to state guidelines and approved by the IACUC of the Sun Yat-sen University Cancer Center. For experiments using human samples, all samples were anonymously coded as stipulated by the Declaration of Helsinki. Written informed consent was obtained from the patients, and the protocol was approved by the IRB of the Sun Yat-sen University Cancer Center.

\section{Author contributions}

$\mathrm{CW}, \mathrm{HN}, \mathrm{ML}$, and $\mathrm{LZ}$ designed the experiments. CW, HN, ML, J. Lin, SL, WZ, and JR performed experiments and analyzed and interpreted the data. JX, WCW, J. Liang, CKS, BW, JC, and MSC provided mice, clinical resources, and technical support. LZ supported and supervised the research. CW and LZ wrote the manuscript, and all authors contributed to editing of the manuscript.

\section{Acknowledgments}

The authors wish to thank Lingyan Zhu and Yunfeng Chen for help with flow cytometry and cell sorting (School of Life Sciences, Sun Yat-sen University). This work was supported by project grants from the National Key R\&D Program of China (2017YFA0505803 and 2018ZX10302205); the National Natural Science Foundation of China (91442205 and 81730044); the Health Medical Collaborative Innovation Program of Guangzhou (201400000001-3); and the Fundamental Research Funds for the Central Universities (16lgjc46 and 171gjc32).

Address correspondence to: Limin Zheng, State Key Laboratory of Oncology in South China, Sun Yat-sen University Cancer Center, Guangzhou 510060, P.R. China. Phone: 86.20.84112163; Email: zhenglm@mail.sysu.edu.cn.
1. McAllister SS, Weinberg RA. The tumourinduced systemic environment as a critical regulator of cancer progression and metastasis. Nat Cell Biol. 2014;16(8):717-727.

2. Kitamura T, Qian BZ, Pollard JW. Immune cell promotion of metastasis. Nat Rev Immunol. 2015;15(2):73-86.

3. Noy R, Pollard JW. Tumor-associated macrophages: from mechanisms to therapy. Immunity.
2014;41(1):49-61.

4. Kumar V, Patel S, Tcyganov E, Gabrilovich DI. The nature of myeloid-derived suppressor cells in the tumor microenvironment. Trends Immunol. 2016;37(3):208-220.

5. Coffelt SB, Wellenstein MD, de Visser KE. Neutrophils in cancer: neutral no more. Nat Rev Cancer. 2016;16(7):431-446.

6. Di Mitri D, et al. Tumour-infiltrating Gr-1+ myeloid cells antagonize senescence in cancer. Nature. 2014;515(7525):134-137.

7. Cui TX, et al. Myeloid-derived suppressor cells enhance stemness of cancer cells by inducing microRNA101 and suppressing the corepressor CtBP2. Immunity. 2013;39(3):611-621.

8. Talmadge JE, Gabrilovich DI. History of myeloid-derived suppressor cells. Nat Rev Cancer. 2013;13(10):739-752. 
9. Kitamura T, et al. CCL2-induced chemokine cascade promotes breast cancer metastasis by enhancing retention of metastasis-associated macrophages. JExp Med. 2015;212(7):1043-1059.

10. Powell DR, Huttenlocher A. Neutrophils in the Tumor Microenvironment. Trends Immunol. 2016;37(1):41-52.

11. Condamine T, et al. ER stress regulates myeloid-derived suppressor cell fate through TRAIL-R-mediated apoptosis. J Clin Invest. 2014;124(6):2626-2639.

12. Casbon AJ, et al. Invasive breast cancer reprograms early myeloid differentiation in the bone marrow to generate immunosuppressive neutrophils. Proc Natl Acad Sci U S A. 2015;112(6):E566-E575.

13. Strauss L, et al. RORC1 Regulates Tumor-Promoting "Emergency" Granulo-Monocytopoiesis. Cancer Cell. 2015;28(2):253-269.

14. Gomez D, et al. Preoperative neutrophil-to-lymphocyte ratio as a prognostic predictor after curative resection for hepatocellular carcinoma. World J Surg. 2008;32(8):1757-1762.

15. Walsh SR, Cook EJ, Goulder F, Justin TA, Keeling NJ. Neutrophil-lymphocyte ratio as a prognostic factor in colorectal cancer. J Surg Oncol. 2005;91(3):181-184.

16. Wu WC, et al. Circulating hematopoietic stem and progenitor cells are myeloid-biased in cancer patients. Proc Natl Acad Sci U S A. 2014;111(11):4221-4226.

17. Marigo I, et al. Tumor-induced tolerance and immune suppression depend on the $\mathrm{C}$ / EBPbeta transcription factor. Immunity. 2010;32(6):790-802.

18. Pylayeva-Gupta Y, Lee KE, Hajdu CH, Miller G, Bar-Sagi D. Oncogenic Kras-induced GM-CSF production promotes the development of pancreatic neoplasia. Cancer Cell. 2012;21(6):836-847.

19. Bayne LJ, et al. Tumor-derived granulocyte-macrophage colony-stimulating factor regulates myeloid inflammation and $\mathrm{T}$ cell immunity in pancreatic cancer. Cancer Cell. 2012;21(6):822-835.

20. Cortez-Retamozo V, et al. Angiotensin II drives the production of tumor-promoting macrophages. Immunity. 2013;38(2):296-308.

21. Cortez-Retamozo V, et al. Origins of tumor-associated macrophages and neutrophils. Proc Natl Acad Sci U S A. 2012;109(7):2491-2496.

22. Bronte V, Pittet MJ. The spleen in local and systemic regulation of immunity. Immunity. 2013;39(5):806-818

23. Darlington GJ, Bernhard HP, Miller RA, Ruddle FH. Expression of liver phenotypes in cultured mouse hepatoma cells. J Natl Cancer Inst. 1980;64(4):809-819.

24. Darlington GJ. Liver cell lines. Meth Enzymol. 1987;151:19-38.

25. Osawa M, Hanada K, Hamada H, Nakauchi H. Long-term lymphohematopoietic reconstitution by a single CD34-low/negative hematopoietic stem cell. Science. 1996;273(5272):242-245.
26. Oguro H, Ding L, Morrison SJ. SLAM family markers resolve functionally distinct subpopulations of hematopoietic stem cells and multipotent progenitors. Cell Stem Cell. 2013;13(1):102-116.

27. Kiel MJ, Yilmaz OH, Iwashita T, Yilmaz OH, Terhorst C, Morrison SJ. SLAM family receptors distinguish hematopoietic stem and progenitor cells and reveal endothelial niches for stem cells. Cell. 2005;121(7):1109-1121.

28. McCulloch EA. CFU-S : An Assay for Pluripotent Myelopoietic Stem Cells. Methods Mol Med. 2002;63:153-160.

29. Akashi K, Traver D, Miyamoto T, Weissman IL. A clonogenic common myeloid progenitor that gives rise to all myeloid lineages. Nature. 2000;404(6774):193-197.

30. Zhao JL, et al. Conversion of danger signals into cytokine signals by hematopoietic stem and progenitor cells for regulation of stress-induced hematopoiesis. Cell Stem Cell. 2014;14(4):445-459.

31. Condamine T, Mastio J, Gabrilovich DI. Transcriptional regulation of myeloid-derived suppressor cells. JLeukoc Biol. 2015;98(6):913-922.

32. Condamine T, Gabrilovich DI. Molecular mechanisms regulating myeloid-derived suppressor cell differentiation and function. Trends Immunol. 2011;32(1):19-25.

33. Inra CN, et al. A perisinusoidal niche for extramedullary haematopoiesis in the spleen. Nature. 2015;527(7579):466-471.

34. Dutta P, et al. Myocardial Infarction Activates CCR2(+) Hematopoietic Stem and Progenitor Cells. Cell Stem Cell. 2015;16(5):477-487.

35. Yu W, Choi GS, Chung HY. Randomized clinical trial of splenectomy versus splenic preservation in patients with proximal gastric cancer. Br J Surg. 2006;93(5):559-563.

36. Motomura T, et al. Neutrophil-lymphocyte ratio reflects hepatocellular carcinoma recurrence after liver transplantation via inflammatory microenvironment. J Hepatol. 2013;58(1):58-64.

37. Cadili A, de Gara C. Complications of splenectomy. Am J Med. 2008;121(5):371-375.

38. Tang H, et al. PD-L1 on host cells is essential for PD-L1 blockade-mediated tumor regression. J Clin Invest. 2018;128(2):580-588.

39. Lin H, et al. Host expression of PD-L1 determines efficacy of PD-L1 pathway blockade-mediated tumor regression. J Clin Invest. 2018;128(2):805-815.

40. Lennartsson J, Rönnstrand L. Stem cell factor receptor/c-Kit: from basic science to clinical implications. Physiol Rev. 2012;92(4):1619-1649.

41. Yin AH, et al. AC133, a novel marker for human hematopoietic stem and progenitor cells. Blood. 1997;90(12):5002-5012.

42. Ugel S, et al. Immune tolerance to tumor antigens occurs in a specialized environment of the spleen. Cell Rep. 2012;2(3):628-639.

43. Baldridge MT, King KY, Boles NC, Weksberg DC, Goodell MA. Quiescent haematopoi- etic stem cells are activated by IFN-gamma in response to chronic infection. Nature. 2010;465(7299):793-797.

44. Si Y, Tsou CL, Croft K, Charo IF. CCR2 mediates hematopoietic stem and progenitor cell trafficking to sites of inflammation in mice. J Clin Invest. 2010;120(4):1192-1203.

45. Griseri T, McKenzie BS, Schiering C, Powrie F. Dysregulated hematopoietic stem and progenitor cell activity promotes interleukin-23-driven chronic intestinal inflammation. Immunity. 2012;37(6):1116-1129.

46. Leuschner F, et al. Rapid monocyte kinetics in acute myocardial infarction are sustained by extramedullary monocytopoiesis. J Exp Med. 2012;209(1):123-137.

47. Murphy AJ, et al. ApoE regulates hematopoietic stem cell proliferation, monocytosis, and monocyte accumulation in atherosclerotic lesions in mice. J Clin Invest. 2011;121(10):4138-4149.

48. Dutta $P$, et al. Myocardial infarction accelerates atherosclerosis. Nature. 2012;487(7407):325-329.

49. Levy L, Mishalian I, Bayuch R, Zolotarov L, Michaeli J, Fridlender ZG. Splenectomy inhibits non-small cell lung cancer growth by modulating anti-tumor adaptive and innate immune response. Oncoimmunology. 2015;4(4):e998469.

50. Shand FH, et al. Tracking of intertissue migration reveals the origins of tumor-infiltrating monocytes. Proc Natl Acad Sci U S A. 2014;111(21):7771-7776.

51. Chen DS, Mellman I. Oncology meets immunology: the cancer-immunity cycle. Immunity. 2013;39(1):1-10.

52. Sharma P, Hu-Lieskovan S, Wargo JA, Ribas A. Primary, Adaptive, and Acquired Resistance to Cancer Immunotherapy. Cell. 2017;168(4):707-723.

53. Baumeister SH, Freeman GJ, Dranoff G, Sharpe AH. Coinhibitory pathways in immunotherapy for cancer. Annu Rev Immunol. 2016;34:539-573.

54. Vanneman M, Dranoff G. Combining immunotherapy and targeted therapies in cancer treatment. Nat Rev Cancer. 2012;12(4):237-251.

55. Xiao X, et al. PD-1hi identifies a novel regulatory B-cell population in human hepatoma that promotes disease progression. Cancer Discov. 2016;6(5):546-559.

56. Kuang DM, et al. B7-H1-expressing antigen-presenting cells mediate polarization of protumorigenic Th22 subsets. JClin Invest. 2014;124(10):4657-4667.

57. Till JE, McCulloch EA. A direct measurement of the radiation sensitivity of normal mouse bone marrow cells. Radiat Res. 1961;14:213-222.

58. Becker AJ, McCulloch EA, Till JE. Cytological demonstration of the clonal nature of spleen colonies derived from transplanted mouse marrow cells. Nature. 1963;197:452-454

59. Dolcetti L, Peranzoni E, Bronte V. Measurement of myeloid cell immune suppressive activity. Curr Protoc Immunol. 2010; Chapter 14:Unit 14.17. 